\title{
Homogenization for non-local elliptic operators in both perforated and non-perforated domains
}

\author{
Loredana Bălilescu* Amrita Ghosh ${ }^{\dagger} \quad$ Tuhin Ghosh
}

\begin{abstract}
In this paper, we focus on the homogenization process of the non-local elliptic boundary value problem

$$
\mathcal{L}_{\varepsilon}^{s} u_{\varepsilon}=\left(-\nabla \cdot\left(A_{\varepsilon}(x) \nabla\right)\right)^{s} u_{\varepsilon}=f \text { in } \mathcal{O},
$$

with $0<s<1$, considering non-homogeneous Dirichlet type condition outside of the bounded domain $\mathcal{O} \subseteq \mathbb{R}^{n}$. We find the homogenized problem by using the $H$-convergence method, as $\varepsilon \rightarrow 0$, under standard uniform ellipticity, boundedness and symmetry assumptions on coefficients $A_{\varepsilon}(x)$, with the homogenized coefficients as the standard $H$-limit (cf. 22]) of the sequence $\left\{A_{\varepsilon}\right\}_{\varepsilon>0}$. We also prove that the commonly referred to as the strange term in the literature (see [10, Chapter 4]) does not appear in the homogenized problem associated with the fractional Laplace operator $(-\Delta)^{s}$ in a perforated domain. Both of these results have been obtained in the class of general microstructures. Consequently, we could certify that the homogenization process, as $\varepsilon \rightarrow 0$, is stable under $s \rightarrow 1^{-}$in the non-perforated domains, but not necessarily in the case of perforated domains.
\end{abstract}

Keywords: Homogenization, $H$-convergence, non-local operators, integro-differential operator, fractional operators.

\section{Introduction}

The general question tackled in this paper is the homogenization process of Dirichlet type problem associated with fractional elliptic non-local operator in bounded domains. Precisely, let $\mathcal{L}=-\nabla \cdot(A(x) \nabla)$ be the classical uniformly elliptic operator in divergence form with the anisotropic matrix valued function $A(x)$ defined in whole space $\mathbb{R}^{n}$. Then, for $0<s<1$, we consider the fractional non-local operator (for the definition, see Section 2 below):

$$
\mathcal{L}^{s}=(-\nabla \cdot(A(x) \nabla))^{s} .
$$

We are interested in the restriction of the fractional Laplacian $\mathcal{L}^{\mathcal{s}}$ in a bounded domain $\mathcal{O} \subset \mathbb{R}^{n}$ and the associated non-homogeneous Dirichlet exterior boundary value problem $\mathcal{L}^{s} u=f$ in a smooth enough bounded domain $\mathcal{O} \subset \mathbb{R}^{n}$ with $u=g$ in $\mathbb{R}^{n} \backslash \mathcal{O}$.

*Department of Mathematics and Computer Science, University of Piteşti, 110040 Piteşti, Str. Târgu din Vale nr.1, Argeş, Romania and Department of Mathematics, Federal University of Santa Catarina, Brasil; smaranda@dim.uchile.cl

$\dagger$ Department of Mathematics, Université de Pau et des Pays de l'Adour, France amrita.ghosh@univ-pau.fr

$\ddagger$ Department of Mathematics, University of Washington; tuhing@uw.edu 
These kind of fractional and non-local operators often arise in problems modelling diffusion process, ergodic random environments and random processes with jumps, enabling possible applications in probability theory, physics, finance, and biology, to name a few (for more details, see the survey works [7, 27]). In particular, the above operator $\mathcal{L}^{s}$ as a linear integro-differential operator (see (2.27) ) could be considered as an infinitesimal generator of generalized Lévy processes of the probabilistic/stochastic model under consideration with a random process that allows long jumps with a polynomial tail (see the books [2, 6, 13]). For example, if $g=0$, probabilistically it represents the infinitesimal generator of a symmetric $2 s$-stable Lévy process that particles are killed upon leaving the domain $\mathcal{O}$.

The paper aims at providing a macro scale approximation to a problem with heterogeneities/microstructures at micro scale $\varepsilon$ by suitably averaging out small scales $(\varepsilon \rightarrow 0)$ and by incorporating their effects on large scales. These effects are quantified by the so-called homogenized coefficients [1, 5, 18, 35]. We will be using the $H$-convergence method (for more details on $H$-limits, we refer to [1, 23, 35]), under standard uniform ellipticity, boundedness and symmetric assumptions on the coefficient matrices $\left\{A_{\varepsilon}(x)\right\}_{\varepsilon>0}$.

More precisely, let us consider $s \in(0,1), \mathcal{O} \subset \mathbb{R}^{n}$ be a bounded Lipschitz domain. For each $\varepsilon>0$, consider $u_{\varepsilon} \in H^{s}\left(\mathbb{R}^{n}\right)$, which is the solution to the following non-local Dirichlet type problem:

$$
\begin{cases}\mathcal{L}_{\varepsilon}^{s} u_{\varepsilon}=\left(-\nabla \cdot\left(A_{\varepsilon}(x) \nabla\right)\right)^{s} u_{\varepsilon}=f & \text { in } \mathcal{O}, \\ u_{\varepsilon}=g & \text { in } \mathbb{R}^{n} \backslash \mathcal{O},\end{cases}
$$

for some $f \in \widetilde{H}^{s}(\mathcal{O})^{*}$ (see 2.31) below for the definition of this space) and $g \in H^{s}\left(\mathbb{R}^{n}\right)$.

Our main goal is to pass to the limit in the above problem (1.1), as $\varepsilon \rightarrow 0$, and to find the limit equation or the homogenized problem. Our main finding is that the homogenized equation is governed by the non-local elliptic operator

$$
\mathcal{L}_{*}^{s}=\left(-\nabla \cdot A_{*}(x) \nabla\right)^{s},
$$

where $A_{*}(x)$ is the standard $H$-limit of the sequence $\left\{A_{\varepsilon}(x)\right\}_{\varepsilon>0}$ in $\mathbb{R}^{n}$ under the standard uniform ellipticity and boundedness hypotheses on $\left\{A_{\varepsilon}(x)\right\}_{\varepsilon>0}=\left\{\left(a_{\varepsilon}^{i j}(x)\right)_{1 \leq i, j \leq n}\right\}_{\varepsilon>0}$ given as

$$
\left\{\begin{array}{l}
a_{\varepsilon}^{i j}(x)=a_{\varepsilon}^{j i}(x) \text { for all } x \in \mathbb{R}^{n}, 1 \leq i, j \leq n, \text { and } \\
\lambda^{-1}|\xi|^{2} \leq \sum_{i, j=1}^{n} a_{\varepsilon}^{i j}(x) \xi_{i} \xi_{j} \leq \lambda|\xi|^{2} \text { for all } x \in \mathbb{R}^{n}, \varepsilon>0, \text { and for some } \lambda>0
\end{array}\right.
$$

Let us state our first main result concerning the homogenization process for fractional nonlocal elliptic operators in non-perforated domain.

Theorem 1.1. Let $s \in(0,1), \mathcal{O} \subset \mathbb{R}^{n}$ be a bounded domain with sufficiently smooth boundary. We assume that the sequence $\left\{A_{\varepsilon}(x)\right\}_{\varepsilon>0}$ satisfies condition 1.2 . For each $\varepsilon>0$, let $u_{\varepsilon} \in$ $H^{s}\left(\mathbb{R}^{n}\right)$ be the solution of problem (1.1), for some fixed $f \in \widetilde{H^{s}(\mathcal{O})^{*}}$ and $g \in H^{s}\left(\mathbb{R}^{n}\right)$. Then, as $\varepsilon \rightarrow 0$, up to a subsequence, we have

$$
u_{\varepsilon} \rightarrow u \text { weakly in } H^{s}\left(\mathbb{R}^{n}\right),
$$

with the limit $u \in H^{s}\left(\mathbb{R}^{n}\right)$ characterized as the unique solution of the following homogenized problem:

$$
\begin{cases}\mathcal{L}_{*}^{s} u=\left(-\nabla \cdot\left(A_{*}(x) \nabla\right)\right)^{s} u=f & \text { in } \mathcal{O}, \\ u=g & \text { in } \mathbb{R}^{n} \backslash \mathcal{O},\end{cases}
$$

where $A_{*}(x)$ is the $H$-limit of the sequence $\left\{A_{\varepsilon}(x)\right\}_{\varepsilon>0}$ in $\mathbb{R}^{n}$, that is,

$$
A_{\varepsilon} \nabla w_{\varepsilon} \rightarrow A_{*} \nabla w \quad \text { weakly in } L^{2}\left(\mathbb{R}^{n}\right)^{n},
$$


for all test sequences $w_{\varepsilon} \in H^{1}\left(\mathbb{R}^{n}\right)$ satisfying

$$
\begin{gathered}
w_{\varepsilon} \rightarrow w \quad \text { weakly in } H^{1}\left(\mathbb{R}^{n}\right), \\
-\nabla \cdot\left(A_{\varepsilon} \nabla w_{\varepsilon}\right) \quad \text { strongly convergent in } H^{-1}\left(\mathbb{R}^{n}\right) .
\end{gathered}
$$

Moreover, we have the following flux and energy convergences, respectively, as $\varepsilon \rightarrow 0$ :

$$
\begin{array}{r}
\mathcal{L}_{\varepsilon}^{s / 2} u_{\varepsilon} \rightarrow \mathcal{L}_{*}^{s / 2} u \quad \text { weakly in } L^{2}\left(\mathbb{R}^{n}\right), \\
\left\|\mathcal{L}_{\varepsilon}^{s / 2} u_{\varepsilon}\right\|_{L^{2}\left(\mathbb{R}^{n}\right)} \rightarrow\left\|\mathcal{L}_{*}^{s / 2} u\right\|_{L^{2}\left(\mathbb{R}^{n}\right)} .
\end{array}
$$

Remark 1.1. The above result is also applicable for spectral non-local operator $\mathcal{L}_{S}^{s}$, which is defined by the normalized eigenfunctions and eigenvalues for the operator $\mathcal{L}$ in $\mathcal{O}$ with homogeneous Dirichlet/Neumann boundary conditions. Let $\left\{\varphi_{k}\right\}_{k \in \mathbb{N}}$ denotes an orthonormal basis of $L^{2}(\mathcal{O})$ satisfying

$$
\begin{cases}\mathcal{L} \varphi_{k}=\left(-\nabla \cdot(A(x) \nabla) \varphi_{k}=\lambda_{k} \varphi_{k}\right. & \text { in } \mathcal{O}, \\ \varphi_{k}=0 & \text { on } \partial \mathcal{O} .\end{cases}
$$

Then, the spectral non-local operator $\mathcal{L}_{S}^{s}(0<s<1)$ is defined as

$$
\forall u \in H^{s}(\mathcal{O}), \quad \mathcal{L}_{S}^{s} u=\sum_{k=1}^{\infty} \lambda_{k}^{s}\left\langle\varphi_{k}, u\right\rangle_{2} \varphi_{k} \quad \text { in } \mathcal{O} .
$$

In [9] (also [8]) the Caffarelli-Stinga result (see Proposition 3.1, 3.2 for a statement of the result) was proved for this operator, thus achieving a local problem posed on a semi-infinite cylinder $\mathcal{O} \times(0, \infty)$, whose Dirichlet-Neumann map defines the operator $\mathcal{L}_{S}^{s}$. Since our method to prove Theorem 1.1 relies on the analysis of the extended local problem in $\mathbb{R}_{+}^{n+1}$ (as shown in Section 3), we say the above theorem holds true for the homogenization of the spectral non-local operators $\left\{\left(\mathcal{L}_{S}^{s}\right)_{\varepsilon}\right\}_{\varepsilon>0}$ in $\mathcal{O}$.

Let us now introduce our second problem to be considered and state the second main result. To this end, we define a sequence of any closed subsets $\left\{T_{\varepsilon}\right\}_{\varepsilon>0} \subset \mathbb{R}^{n}$, which are called holes, and we take the perforated domain $\mathcal{O}_{\varepsilon}$ simply defined as follows:

$$
\mathcal{O}_{\varepsilon}:=\mathcal{O} \backslash \underset{0<\delta \leq \varepsilon}{\cup} T_{\delta}
$$

with the condition on Lebesgue measure:

$$
\lim _{\varepsilon \rightarrow 0}\left|\mathcal{O} \backslash \mathcal{O}_{\varepsilon}\right|=0
$$

For $s \in(0,1)$ and for each $\varepsilon>0$, let $u_{\varepsilon} \in H^{s}\left(\mathbb{R}^{n}\right)$ be the solution of the following non-local Dirichlet problem in a perforated domain:

$$
\begin{cases}(-\Delta)^{s} u_{\varepsilon}=f & \text { in } \mathcal{O}_{\varepsilon} \\ u_{\varepsilon}=g & \text { in } \mathbb{R}^{n} \backslash \mathcal{O}_{\varepsilon}\end{cases}
$$

for some $f \in \widetilde{H}^{s}(\mathcal{O})^{*}$ and $g \in H^{s}\left(\mathbb{R}^{n}\right)$.

Motivated from the first result (cf. Theorem 1.1) we allow such perforated domains $\mathcal{O}_{\varepsilon}$ where the following hypothesis are satisfied. Let us assume that there exist a sequence of functions $\left\{w_{\varepsilon}\right\}_{\varepsilon>0}$ such that:

(H1) $w_{\varepsilon} \in H^{1}(\mathcal{O})$; 
(H2) $w_{\varepsilon}=0$ on the holes $\underset{0<\delta \leq \varepsilon}{\cup} T_{\delta}$;

(H3) $w_{\varepsilon} \rightarrow 1$ weakly in $H^{1}(\mathcal{O})$.

We now state our second main result and we show that the commonly referred to as the 'strange term' in the literature does not appear in the homogenized problem associated with the fractional Laplace operator in a perforated domain.

Theorem 1.2. Let $s \in(0,1), \mathcal{O} \subset \mathbb{R}^{n}$ be a bounded domain with sufficiently smooth boundary and $\left\{\mathcal{O}_{\varepsilon}\right\}_{\varepsilon>0}$ be defined by (1.5) with 1.6. We assume the above hypothesis (H1)-(H3) on $\left\{\mathcal{O}_{\varepsilon}\right\}_{\varepsilon>0}$. For each $\varepsilon>0$, let $u_{\varepsilon} \in H^{s}\left(\mathbb{R}^{n}\right)$ be the solution of problem (1.7) for given $f \in \widetilde{H}^{s}(\mathcal{O})^{*}$ and $g \in H^{s}\left(\mathbb{R}^{n}\right)$. Then, as $\varepsilon \rightarrow 0$, up to a subsequence, we have

$$
u_{\varepsilon} \rightarrow u \quad \text { weakly in } H^{s}\left(\mathbb{R}^{n}\right) \text {, }
$$

where the limit $u \in H^{s}\left(\mathbb{R}^{n}\right)$ can be characterized as the unique solution of the following homogenized problem:

$$
\begin{cases}(-\Delta)^{s} u=f & \text { in } \mathcal{O}, \\ u=g & \text { in } \mathbb{R}^{n} \backslash \mathcal{O} .\end{cases}
$$

Remark 1.2. Since $\mathcal{L}^{s} w \rightarrow \mathcal{L} w$ in $L^{2}(\mathcal{O})$, as $s \rightarrow 1^{-}$, for $w \in H^{2}(\mathcal{O})$ (see [16, 11]), then from our result Theorem 1.1 we can essentially claim that the homogenization process, as $\varepsilon \rightarrow 0$, is stable, under the limiting approach as $s \rightarrow 1^{-}$, that is, both of these limit operations, as $\varepsilon \rightarrow 0$ and as $s \rightarrow 1^{-}$, are interchangeable. However, in Theorem 1.2. we find out it is not the case. Both limiting processes, as $\varepsilon \rightarrow 0$ and as $s \rightarrow 1^{-}$, may not be always interchangeable because, in the local case, depending on the estimated size of the tiny holes $\left\{T_{\varepsilon}\right\}_{\varepsilon>0}$, one might end up having some nonzero zeroth order extra term (say $\left.\mu(x)\right)$ commonly referred to as a "strange term" with the Laplacian in the homogenized operator as $-\Delta+\mu$ (see [10, Chapter 4], [12]).

The above homogenization results are new in the non-local settings and also help to provide a certain classification of perforated and non-perforated domains with respect to the fractional power of an elliptic operator. In both Theorem 1.1 and Theorem 1.2 we do not assume any periodicity or scaling conditions, neither on the sequence $\left\{A_{\varepsilon}(x)\right\}_{\varepsilon>0}$ in the nonperforated case, nor on the sequence of perforated domains $\left\{\mathcal{O}_{\varepsilon}\right\}_{\varepsilon>0}$ respectively, in order to study the homogenization process.

Let us now review few known studies of homogenization problems in non-local settings. The known cases are mostly in some prototype of integro-differential operator. For example, a non-local linear operator with a kernel of convolution type in periodic medium [25], concerning certain diffusion process with jumps have been considered. That is also known Feller process generated by an integro-differential operator [30]. Homogenization of a certain class of integrodifferential equations with Lévy operators [3], including scaling limits for symmetric Itô-Lévy processes in random medium [26] has been studied. Additionally, homogenization of a large class of fully non-linear elliptic integro-differential equations in periodic medium can be found in [31. For example, one prototype of such integro-differential operator under consideration in $[25]$ is

$$
\mathcal{L}^{\varepsilon} u_{\varepsilon}=\varepsilon^{-n-2} \lambda\left(\frac{x}{\varepsilon}\right) \int_{\mathbb{R}^{n}} a\left(\frac{x-y}{\varepsilon}\right) \mu\left(\frac{y}{\varepsilon}\right)\left(u_{\varepsilon}(y)-u_{\varepsilon}(x)\right) d y,
$$

where $\lambda, \mu$ are bounded positive periodic functions characterizing the properties of the medium, and $a$ is the jump kernel being a symmetric positive integrable function. They obtain the limit operator as a local operator $\mathcal{L}=-\sum_{i, j=1}^{n} \Theta_{i j} \frac{\partial^{2} u}{\partial x_{i} \partial x_{j}}$, where the homogenized coefficient 
$\Theta_{i j}$ can be derived from $a, \lambda, \mu$. In [38, the author considers the stochastic homogenization for elliptic integro-differential equations modelling stationary ergodic random environments:

$F_{\varepsilon}\left(u_{\varepsilon}, \frac{x}{\varepsilon}, \omega\right)=\inf _{\alpha} \sup _{\beta}\left\{f^{\alpha \beta}\left(\frac{x}{\varepsilon}, \omega\right)+\int_{\mathbb{R}^{n}}\left(u_{\varepsilon}(x+y)+u_{\varepsilon}(x-y)-2 u_{\varepsilon}(x)\right) K^{\alpha \beta}\left(\frac{x}{\varepsilon}, y, \omega\right) d y\right\}$.

Under some suitable conditions over the kernel $K^{\alpha \beta}$, the author obtains the homogenized equation as the certain viscosity solution of a non-local, elliptic, and translation invariant operator of the same form above. However, in this paper, we don't restrict ourselves in certain examples and rather move into considering classical non-local elliptic problems in a bounded domain including both perforated and non-perforated types and study the homogenization process.

The outline of the remaining paper is the following. Section 2 deals with the functional framework of the fractional non-local elliptic operators $\mathcal{L}^{s}$. In Section 3 we introduce an extension problem which characterize this non-local operator $\mathcal{L}^{s}$. In Section 4 we give the proof of our first main result. Finally, Section 5 focuses on the homogenization process of the fractional Laplace operator in perforated domains.

\section{Functional framework of the fractional non-local elliptic operator}

Let us consider $\left\{\mathcal{L}_{\varepsilon}\right\}_{\varepsilon>0}$ a sequence of linear second order partial differential operator of the divergence form defined in the entire space $\mathbb{R}^{n}$ as follows:

$$
\mathcal{L}_{\varepsilon}:=-\nabla \cdot\left(A_{\varepsilon}(x) \nabla\right),
$$

where $\left\{A_{\varepsilon}(x)\right\}_{\varepsilon>0}=\left\{\left(a^{i j}\left(\frac{x}{\varepsilon}\right)\right)\right\}_{\varepsilon>0}, x \in \mathbb{R}^{n}$, is a sequence of $n \times n$ symmetric matrices satisfying the uniform ellipticity conditions 1.2 . We are going to study the sequence of operators $\left\{\mathcal{L}_{\varepsilon}^{s}\right\}_{\varepsilon>0}$, with $0<s<1$,

$$
\mathcal{L}_{\varepsilon}^{s}:=\left(-\nabla \cdot\left(A_{\varepsilon}(x) \nabla\right)\right)^{s},
$$

defined over the entire space $\mathbb{R}^{n}$ and which will be completely defined in the sequel.

Let us now consider the following differential equation associated with this operator in the bounded domain $\mathcal{O}$ :

$$
\mathcal{L}_{\varepsilon}^{s} u_{\varepsilon}=f \quad \text { in } \mathcal{O},
$$

for some suitable $f$. Next, in order to have a well-posed Dirichlet problem, we assume some exterior boundary condition as follows:

$$
u_{\varepsilon}=g \quad \text { in } \mathbb{R}^{n} \backslash \mathcal{O}
$$

for some suitable $g$. Thus, the homogenization problem that we study is the following Dirichlet problem:

$$
\begin{aligned}
\mathcal{L}_{\varepsilon}^{s} u_{\varepsilon}=f & & \text { in } \mathcal{O}, \\
u_{\varepsilon}=g & & \text { in } \mathbb{R}^{n} \backslash \mathcal{O} .
\end{aligned}
$$

Let us denote by $\mathcal{L}$ a second order linear elliptic operator in the divergence form

$$
\mathcal{L}:=-\nabla \cdot(A(x) \nabla)
$$


which is defined in the entire space $\mathbb{R}^{n}$ for $n \geq 2$, where $A(x)=\left(a_{i j}(x)\right)_{i, j}, x \in \mathbb{R}^{n}$ is an $n \times n$ symmetric matrix satisfying the symmetry and ellipticity conditions 1.2 . We also assume that the variable coefficients of $\mathcal{L}$ are enough regular, precisely

$$
a^{i j}=a^{j i} \in C^{2}\left(\mathbb{R}^{n}\right), \quad 1 \leq i, j \leq n .
$$

It is well known that the operator $\mathcal{L}$ together with the domain

$$
\operatorname{Dom}(\mathcal{L})=H^{2}\left(\mathbb{R}^{n}\right)
$$

is the maximal extension such that $\mathcal{L}$ is self-adjoint and densely defined in $L^{2}\left(\mathbb{R}^{n}\right.$ ) (see, for instance, [16]).

\subsection{Fractional Sobolev spaces and non-local elliptic fractional differential operator}

In this subsection, we will introduce the variable coefficients fractional non-local operator $\mathcal{L}^{s}=(-\nabla \cdot(A(x) \nabla))^{s}$. Let us note that, for $A(x)$ being an identity matrix, the operator $\mathcal{L}^{s}$ becomes the well-known fractional Laplace operator $(-\Delta)^{s}$, which has been widely studied in papers [8, 9, 29, 32] and the references therein.

In this paper, we denote by $C$ a general constant that may change in each occurrence and which will depend on the parameters involved. Wherever it is necessary, we are going to point out the dependence of $C$ on the parameters. Moreover, $\Gamma$ stands for the Gamma function in the rest of the paper.

Let us restrict our attention to the case $0<s<1$. In this interval, we have that $\Gamma(-s):=\frac{\Gamma(1-s)}{-s}<0$.

\section{Spectral approach of non-local elliptic fractional differential operator}

We begin by defining the fractional operator $\mathcal{L}^{s}$ with $s \in(0,1)$, via the spectral characterization of $\mathcal{L}$ (for more details, see [16, 24, 28, 34]). Suppose that $\mathcal{L}$ is a linear second order differential self-adjoint operator which is nonnegative and densely defined on $L^{2}\left(\mathbb{R}^{n}\right)$ for $n \geq 2$. There is a unique resolution $E$ of the identity, supported on the spectrum of $\mathcal{L}$ which is a subset of $[0, \infty)$, such that

$$
I=\int_{0}^{\infty} d E(\lambda)
$$

and

$$
\mathcal{L}:=\int_{0}^{\infty} \lambda d E(\lambda)
$$

that is,

$$
\langle\mathcal{L} f, g\rangle_{L^{2}\left(\mathbb{R}^{n}\right)}:=\int_{0}^{\infty} \lambda d E_{f, g}(\lambda), f \in \operatorname{Dom}(\mathcal{L}), g \in L^{2}\left(\mathbb{R}^{n}\right),
$$

where $d E_{f, g}(\lambda)$ is a regular Borel complex measure of bounded variation concentrated on the spectrum of $\mathcal{L}$, with

$$
\left.d E_{f, g}\right|_{\mid(0, \infty)} \leq\|f\|_{L^{2}\left(\mathbb{R}^{n}\right)}\|g\|_{L^{2}\left(\mathbb{R}^{n}\right)} .
$$

The norm $\|\mathcal{L} f\|_{L^{2}\left(\mathbb{R}^{n}\right)}, f \in \operatorname{Dom}(\mathcal{L})$, is defined as follows:

$$
\|\mathcal{L} f\|_{L^{2}\left(\mathbb{R}^{n}\right)}^{2}:=\int_{0}^{\infty}|\lambda|^{2} d E_{f, f}(\lambda)
$$


If $\phi(\lambda)$ is a real measurable function defined on $[0, \infty)$, then the operator $\phi(\mathcal{L})$ is formally given by

$$
\phi(\mathcal{L})=\int_{0}^{\infty} \phi(\lambda) d E(\lambda)
$$

That is, $\phi(\mathcal{L})$ is the operator with the domain

$$
\operatorname{Dom}(\phi(\mathcal{L}))=\left\{f \in L^{2}\left(\mathbb{R}^{n}\right): \int_{0}^{\infty}|\phi(\lambda)|^{2} d E_{f, f}(\lambda)<\infty\right\}
$$

defined by

$$
\langle\phi(\mathcal{L}) f, g\rangle_{L^{2}\left(\mathbb{R}^{n}\right)}=\int_{0}^{\infty} \phi(\lambda) d E_{f, g}(\lambda)
$$

and

$$
\|\phi(\mathcal{L}) f\|_{L^{2}\left(\mathbb{R}^{n}\right)}^{2}=\int_{0}^{\infty}|\phi(\lambda)|^{2} d E_{f, f}(\lambda)
$$

Following this construction, we can define the fractional operators $\mathcal{L}^{s}, s \in(0,1)$, with the domain $\operatorname{Dom}\left(\mathcal{L}^{s}\right) \supset \operatorname{Dom}(\mathcal{L})$, as follows:

$$
\mathcal{L}^{s}=\int_{0}^{\infty} \lambda^{s} d E(\lambda)=\frac{1}{\Gamma(-s)} \int_{0}^{\infty}\left(e^{-t \mathcal{L}}-\mathrm{Id}\right) \frac{d t}{t^{1+s}}
$$

Here, $e^{-t \mathcal{L}}(t \geq 0)$ is the heat-diffusion semigroup generated by $\mathcal{L}$, with the domain $L^{2}\left(\mathbb{R}^{n}\right)$, defined by

$$
e^{-t \mathcal{L}}=\int_{0}^{\infty} e^{-t \lambda} d E(\lambda)
$$

which enjoys the contraction property in $L^{2}\left(\mathbb{R}^{n}\right)$, that is,

$$
\left\|e^{-t \mathcal{L}} f\right\| \leq\|f\|_{L^{2}\left(\mathbb{R}^{n}\right)} .
$$

Note that, for $f \in \operatorname{Dom}\left(\mathcal{L}^{s}\right) \cap \operatorname{Dom}\left(\mathcal{L}^{s / 2}\right)$, from 2.15) it follows

$$
\left\langle\mathcal{L}^{s} f, f\right\rangle_{L^{2}\left(\mathbb{R}^{n}\right)}=\int_{0}^{\infty} \lambda^{s} d E_{f, f}(\lambda)=\left\|\mathcal{L}^{s / 2} f\right\|_{L^{2}\left(\mathbb{R}^{n}\right)}^{2} .
$$

Moreover, for $f, g \in \operatorname{Dom}\left(\mathcal{L}^{s}\right) \cap \operatorname{Dom}\left(\mathcal{L}^{s / 2}\right)$, we have

$$
\left\langle\mathcal{L}^{s} f, g\right\rangle_{L^{2}\left(\mathbb{R}^{n}\right)}=\left\langle f, \mathcal{L}^{s} g\right\rangle_{L^{2}\left(\mathbb{R}^{n}\right)}=\int_{0}^{\infty} \lambda^{s} d E_{f, g}(\lambda)=\left\langle\mathcal{L}^{s / 2} f, \mathcal{L}^{s / 2} g\right\rangle_{L^{2}\left(\mathbb{R}^{n}\right)},
$$

where we have used for $f \in \operatorname{Dom}\left(\mathcal{L}^{s / 2}\right)$ and $h \in \operatorname{Dom}\left(\mathcal{L}^{s / 2}\right)$ that

$$
\left\langle\mathcal{L}^{s / 2} f, h\right\rangle_{L^{2}\left(\mathbb{R}^{n}\right)}=\int_{0}^{\infty} \lambda^{s / 2} d E_{f, h}(\lambda)
$$

Taking $h=\mathcal{L}^{s / 2} g$ with $g \in \operatorname{Dom}\left(\mathcal{L}^{s / 2}\right)$, we deduce that

$$
d E_{f, h}=\lambda^{s / 2} d E_{f, g} .
$$




\section{Kernel representation of the operator $\mathcal{L}^{s}$}

Let us write the definition given in 2.17) for any $v \in \operatorname{Dom}\left(\mathcal{L}^{s}\right)$ :

$$
\mathcal{L}^{s} v=\frac{1}{\Gamma(-s)} \int_{0}^{\infty}\left(e^{-t \mathcal{L}} v(x)-v(x)\right) \frac{d t}{t^{1+s}} .
$$

We introduce the distributional heat kernel $W_{t}(x, z)$ of $\mathcal{L}$ satisfying: for any $\varphi, \psi \in$ $H^{s}\left(\mathbb{R}^{n}\right)$,

$$
\left(e^{-t \mathcal{L}} \varphi, \psi\right)_{\mathbb{R}^{n}}=\int_{\mathbb{R}^{n}} \int_{\mathbb{R}^{n}} W_{t}(x, z) \varphi(z) \psi(x) d z d x=\left(\varphi, e^{-t \mathcal{L}} \psi\right)_{\mathbb{R}^{n}}, t \geq 0 .
$$

Since $A(x)$ satisfies 1.2$)$ in $\mathbb{R}^{n}$, using [4], it follows that, for some positive constants $c_{1}, c_{2}, c_{3}, c_{4}$ depending on ellipticity and boundness of $A$ and $n$, we have

$$
c_{1} \frac{e^{-\frac{|x-z|^{2}}{c_{2}} t}}{t^{n / 2}} \leq W_{t}(x, z) \leq c_{3} \frac{e^{-\frac{|x-z|^{2}}{c_{4}} t}}{t^{n / 2}} .
$$

Let us now define the kernel of the heat semi-group $e^{-t \mathcal{L}}$ by

$$
\mathcal{K}^{s}(x, z)=\frac{1}{2|\Gamma(-s)|} \int_{0}^{\infty} W_{t}(x, z) \frac{d t}{t^{1+s}} .
$$

Since $e^{-t \mathcal{L}}$ is symmetric, we get $\mathcal{K}^{s}(x, z)=\mathcal{K}^{s}(z, x)$ for any $x, z \in \mathbb{R}^{n}$, then from 9 , Theorem 2.4 ] it follows that: for all $v, w \in \operatorname{Dom}\left(\mathcal{L}^{s}\right)$,

$$
\left(\mathcal{L}^{s} v, w\right)_{\mathbb{R}^{n}}=\int_{\mathbb{R}^{n}} \int_{\mathbb{R}^{n}}(v(x)-v(z))(w(x)-w(z)) \mathcal{K}^{s}(x, z) d x d z .
$$

Furthermore, a direct computation and using estimate 2.23 on $W_{t}$, one can prove that the kernel $\mathcal{K}^{s}$ enjoys the following pointwise estimate:

$$
c_{1} \frac{\Gamma\left(\frac{n}{2}+s\right)}{2|\Gamma(-s)|} c_{2}^{\frac{n}{2}+s} \frac{1}{|x-z|^{n+2 s}} \leq \mathcal{K}^{s}(x, z) \leq c_{3} \frac{\Gamma\left(\frac{n}{2}+s\right)}{2|\Gamma(-s)|} c_{4}^{\frac{n}{2}+s} \frac{1}{|x-z|^{n+2 s}},
$$

where the constants $c_{1}, c_{2}, c_{3}, c_{4}$ appear in (2.23) and are dependent on the ellipticity and boundness of $A$ and on $n$.

We may also write for $v \in \operatorname{Dom}\left(\mathcal{L}^{s}\right)$ (for more details, see [15]):

$$
\mathcal{L}^{s} v(x)=\mathrm{P} . \mathrm{V} \cdot \int_{\mathbb{R}^{n}}(v(x)-v(z)) \mathcal{K}^{s}(x, z) d z,
$$

where P.V. stands for the standard principal value operator.

\section{Sobolev spaces}

Let $H^{s}\left(\mathbb{R}^{n}\right)=W^{s, 2}\left(\mathbb{R}^{n}\right)$ for $s \in \mathbb{R}$ the standard Sobolev space with the norm

$$
\|u\|_{H^{s}\left(\mathbb{R}^{n}\right)}=\left\|\langle D\rangle^{s} u\right\|_{L^{2}\left(\mathbb{R}^{n}\right)},
$$

where $\langle\xi\rangle=\left(1+|\xi|^{2}\right)^{\frac{1}{2}}$. Let $m(\xi)$ be an arbitrary $C^{\infty}$-smooth polynomial in $\xi$, and the notation $m(D) u=\mathscr{F}^{-1}\{m(\xi) \hat{u}(\xi)\}$ stands for the Fourier multipliers and $\mathscr{F}$ is the classical Fourier transform given by

$$
\widehat{u}(\xi)=\mathscr{F} u(\xi)=\int_{\mathbb{R}^{n}} e^{-i x \cdot \xi} u(x) d x .
$$


We may and shall consider the following $H^{s}\left(\mathbb{R}^{n}\right)$-norm, for $s \in \mathbb{R}^{+}$:

$$
\|u\|_{H^{s}\left(\mathbb{R}^{n}\right)}^{2}=\|u\|_{L^{2}\left(\mathbb{R}^{n}\right)}^{2}+\left\|(-\Delta)^{s / 2} u\right\|_{L^{2}\left(\mathbb{R}^{n}\right)}^{2} .
$$

Let us observe that the semi-norm $\left\|(-\Delta)^{s / 2} u\right\|_{L^{2}\left(\mathbb{R}^{n}\right)}^{2}$ is expressed as follows:

$$
\left\|(-\Delta)^{s / 2} u\right\|_{L^{2}\left(\mathbb{R}^{n}\right)}^{2}=\left((-\Delta)^{s} u, u\right)_{\mathbb{R}^{n}},
$$

where, for $s \in(0,1)$,

$$
(-\Delta)^{s} u(x)=c_{n, s} \text { P.V. } \int_{\mathbb{R}^{n}} \frac{u(x)-u(y)}{|x-y|^{n+2 s}} d y
$$

and

$$
c_{n, s}=\frac{\Gamma\left(\frac{n}{2}+s\right)}{|\Gamma(-s)|} \frac{4^{s}}{\pi^{n / 2}} .
$$

The space $H^{s}(\mathcal{O})$, with $\mathcal{O} \subset \mathbb{R}^{n}$ being an arbitrary open set, is equipped with the following norm (see [20, Chapter 3]):

$$
\|u\|_{H^{s}(\mathcal{O})}:=\inf \left\{\|w\|_{H^{s}\left(\mathbb{R}^{n}\right)}: w \in H^{s}\left(\mathbb{R}^{n}\right) \text { and }\left.w\right|_{\mathcal{O}}=u\right\} .
$$

Furthermore, by taking $\mathcal{C} \subset \mathbb{R}^{n}$ a closed set such that $\operatorname{int}(\mathcal{C}) \neq \emptyset$, we can define

$$
H_{\mathcal{C}}^{s}=H_{\mathcal{C}}^{s}\left(\mathbb{R}^{n}\right)=\left\{u \in H^{s}\left(\mathbb{R}^{n}\right): \operatorname{supp}(u) \subset \mathcal{C}\right\} .
$$

If $\mathcal{O}$ is a Lipschitz domain, then we have the following space identification (for more details, see [20, 36]): for $s \in \mathbb{R}$,

$$
\begin{aligned}
& \widetilde{H}^{s}(\mathcal{O})=H \frac{s}{\mathcal{O}}\left(\mathbb{R}^{n}\right), \\
& \widetilde{H}^{s}(\mathcal{O})^{*}=H_{\overline{\mathcal{O}}}^{s}\left(\mathbb{R}^{n}\right)^{*}=H^{-s}(\mathcal{O}) \text { and } H^{s}(\mathcal{O})^{*}=H_{\overline{\mathcal{O}}}^{-s}\left(\mathbb{R}^{n}\right)
\end{aligned}
$$

and, for $s \in\left(-\frac{1}{2}, \frac{1}{2}\right)$,

$$
H^{s}(\mathcal{O})=H_{\overline{\mathcal{O}}}^{s}\left(\mathbb{R}^{n}\right)=H_{0}^{s}(\mathcal{O})
$$

\subsection{Dirichlet problem for $\mathcal{L}^{s}$}

We consider the following Dirichlet problem for the non-local operator $\mathcal{L}^{s}$

$$
\begin{cases}\mathcal{L}^{s} u=f & \text { in } \mathcal{O}, \\ u=g & \text { in } \mathcal{O}_{e}=\mathbb{R}^{n} \backslash \mathcal{O},\end{cases}
$$

with $f \in \widetilde{H}^{s}(\mathcal{O})^{*}$ and $g \in H^{s}\left(\mathbb{R}^{n}\right)$.

Let us first observe that for any $v \in H^{s}\left(\mathbb{R}^{n}\right)$ with $s \in(0,1), \mathcal{L}^{s} v$ can be defined as a distribution in $H^{-s}\left(\mathbb{R}^{n}\right)$ by 2.25 as follows:

$$
\begin{array}{r}
\left|\left(\mathcal{L}^{s} v, w\right)_{\mathbb{R}^{n}}\right|=\left|\int_{\mathbb{R}^{n}} \int_{\mathbb{R}^{n}}(v(x)-v(z))(w(x)-w(z)) \mathcal{K}^{s}(x, z) d x d z\right| \\
\leq\left(\int_{\mathbb{R}^{n}} \int_{\mathbb{R}^{n}}|v(x)-v(z)|^{2} \mathcal{K}^{s}(x, z) d x d z\right)^{\frac{1}{2}} \cdot\left(\int_{\mathbb{R}^{n}} \int_{\mathbb{R}^{n}}|w(x)-w(z)|^{2} \mathcal{K}^{s}(x, z) d x d z\right)^{\frac{1}{2}} \\
\leq C\|v\|_{H^{s}\left(\mathbb{R}^{n}\right)}\|w\|_{H^{s}\left(\mathbb{R}^{n}\right)}, \quad(2
\end{array}
$$

for any $w \in H^{s}\left(\mathbb{R}^{n}\right)$. Here, we have used that $\mathcal{K}^{s}(x, z) \geq 0$ for all $x \neq z$ and also the estimate (2.26). 
We then consider the following associated bilinear form of the above non-local problem 2.33: for any $v, w \in H^{s}\left(\mathbb{R}^{n}\right)$,

$$
\mathcal{B}^{s}(v, w):=\int_{\mathbb{R}^{n}} \int_{\mathbb{R}^{n}}(v(x)-v(z))(w(x)-w(z)) \mathcal{K}^{s}(x, z) d x d z .
$$

It is easy to see from estimate (2.34) that the above bilinear form $\mathcal{B}^{s}(\cdot, \cdot)$ is well-defined in $H^{s}\left(\mathbb{R}^{n}\right) \times H^{s}\left(\mathbb{R}^{n}\right)$, i.e.,

$$
\left|\mathcal{B}^{s}(v, w)\right| \leq C\|v\|_{H^{s}\left(\mathbb{R}^{n}\right)}\|w\|_{H^{s}\left(\mathbb{R}^{n}\right)} .
$$

We note that, following (2.19), the bilinear form $\mathcal{B}^{s}$ can be also expressed as follows:

$$
\mathcal{B}^{s}(v, w)=\left\langle\mathcal{L}^{s / 2} v, \mathcal{L}^{s / 2} w\right\rangle_{L^{2}\left(\mathbb{R}^{n}\right)} \quad \forall v, w \in H^{s}\left(\mathbb{R}^{n}\right) .
$$

Thus, we have the following existence result (for the complete proof, we refer the reader to [15]):

Proposition 2.1. Let $\mathcal{O} \subset \mathbb{R}^{n}$ as mentioned above, and $\mathcal{B}^{s}$ is a bilinear form defined in 2.35 , then there is a solution $u \in H^{s}\left(\mathbb{R}^{n}\right)$ such that

$$
\mathcal{B}^{s}(u, w)=\langle f, w\rangle \text { for any } w \in \widetilde{H}^{s}(\mathcal{O}) \text { with } u-g \in \widetilde{H}^{s}(\mathcal{O}),
$$

for any $f \in \widetilde{H}^{s}(\mathcal{O})^{*}$ and $g \in H^{s}\left(\mathbb{R}^{n}\right)$, where $\langle\cdot, \cdot\rangle$ stands for the duality pairing between $\left(\widetilde{H}^{s}\right)^{*}$ and $\widetilde{H}^{s}$.

Since 0 is not the eigenvalue of the problem

$$
\left\{\begin{array}{l}
\mathcal{L}^{s} w=0 \quad \text { in } \mathcal{O} \\
w=0 \quad \text { in } \mathcal{O}_{e}
\end{array}\right.
$$

the above solution $u \in H^{s}\left(\mathbb{R}^{n}\right)$ is unique.

In addition, we have the following estimate:

$$
\|u\|_{H^{s}\left(\mathbb{R}^{n}\right)} \leq C\left(\|f\|_{\widetilde{H}^{s}(\mathcal{O})^{*}}+\|g\|_{H^{s}\left(\mathbb{R}^{n}\right)}\right),
$$

for some constant $C>0$ independent of $f$ and $g$ and depending on the ellipticity and boundedness of $A$ (see (2.23) ) and on the dimension $n$.

Remark 2.1. The solution $u \in H^{s}\left(\mathbb{R}^{n}\right)$ of problem 2.33 does not depend on the value of $g \in H^{s}\left(\mathbb{R}^{n}\right)$ on $\mathcal{O}$, it only depends on $\left.g\right|_{\mathcal{O}_{e}}$. Let $g_{1}, g_{2} \in H^{s}\left(\mathbb{R}^{n}\right)$ be such that $g_{1}-g_{2} \in$ $\widetilde{H}^{s}(\mathcal{O})=H_{\overline{\mathcal{O}}}^{s}$. Denote by $u_{j} \in H^{s}\left(\mathbb{R}^{n}\right)$ the solution of 2.33 with the Dirichlet data $g_{j}$ for each $j=1,2$. It is observed that

$$
\widetilde{u}:=u_{1}-u_{2}=\left(u_{1}-g_{1}\right)-\left(u_{2}-g_{2}\right)+\left(g_{1}-g_{2}\right) \in \widetilde{H}^{s}(\mathcal{O})
$$

and $\mathcal{B}_{q}(\widetilde{u}, v)=0$ for any $v \in \widetilde{H}^{s}(\mathcal{O})$. Thus, by unicity of solution of $(2.33)$ with $g=0$, one has $\widetilde{u}=0$. Therefore, one can actually consider the non-local problem (2.33) with Dirichlet data in the quotient space

$$
X:=H^{s}\left(\mathbb{R}^{n}\right) / H_{\mathcal{O}}^{s} \cong H^{s}\left(\mathcal{O}_{e}\right)
$$

provided that $\mathcal{O}$ is Lipschitz.

Remark 2.2 (Flux estimate). Using (2.37), it follows that

$$
\mathcal{B}^{s}(u, u-g)=\langle f, u-g\rangle,
$$


for $f \in \widetilde{H}^{s}(\mathcal{O})^{*}$ and $g \in H^{s}\left(\mathbb{R}^{n}\right)$. Then, we get

$$
\left\|\mathcal{L}^{s / 2} u\right\|_{L^{2}\left(\mathbb{R}^{n}\right)}^{2}-\left\langle\mathcal{L}^{s / 2} u, \mathcal{L}^{s / 2} g\right\rangle_{L^{2}\left(\mathbb{R}^{n}\right)}=\langle f, u-g\rangle,
$$

which implies that

$$
\frac{1}{2}\left\|\mathcal{L}^{s / 2} u\right\|_{L^{2}\left(\mathbb{R}^{n}\right)}^{2} \leq \frac{1}{2}\left\|\mathcal{L}^{s / 2} g\right\|_{L^{2}\left(\mathbb{R}^{n}\right)}^{2}+\|f\|_{\widetilde{H}^{s}(\mathcal{O})^{*}}\left(\|u\|_{H^{s}\left(\mathbb{R}^{n}\right)}+\|g\|_{H^{s}\left(\mathbb{R}^{n}\right)}\right) .
$$

Then, by using $H^{s}\left(\mathbb{R}^{n}\right)$-estimate $(2.38)$ in the right hand side, we simply obtain

$$
\left\|\mathcal{L}^{s / 2} u\right\|_{L^{2}\left(\mathbb{R}^{n}\right)} \leq C\left(\|f\|_{\widetilde{H}^{s}(\mathcal{O})^{*}}+\|g\|_{H^{s}\left(\mathbb{R}^{n}\right)}\right),
$$

for some constant $C>0$ independent of $f$ and $g$ and depending on the ellipticity and boundness of $A$ and on $n$.

\subsection{Limit analysis of $\left\{u_{\varepsilon}\right\}_{\varepsilon>0}$ as $\varepsilon \rightarrow 0$}

We consider following sequence of non-local operators $\left\{\mathcal{L}_{\varepsilon}^{s}\right\}_{\varepsilon>0}=\left\{\left(-\nabla \cdot\left(A_{\varepsilon}(x) \nabla\right)\right)^{s}\right\}_{\varepsilon>0}$ introduced similar to the operator $\mathcal{L}^{s}$, with the sequence $\left\{A_{\varepsilon}(x)\right\}_{\varepsilon>0}$ satisfying the conditions (1.2) and regularity condition 2.11). For each $\varepsilon>0$, let $u_{\varepsilon} \in H^{s}\left(\mathbb{R}^{n}\right)$ solving

$$
\begin{cases}\mathcal{L}_{\varepsilon}^{s} u_{\varepsilon}=f & \text { in } \mathcal{O}, \\ u_{\varepsilon}=g & \text { in } \mathcal{O}_{e}\end{cases}
$$

for $f \in \widetilde{H}^{s}(\mathcal{O})^{*}$ and $g \in H^{s}\left(\mathbb{R}^{n}\right)$ and satisfying the stability and flux estimates:

$$
\left\|u_{\varepsilon}\right\|_{H^{s}\left(\mathbb{R}^{n}\right)} \leq C\left(\|f\|_{\widetilde{H}^{s}(\mathcal{O})^{*}}+\|g\|_{H^{s}\left(\mathbb{R}^{n}\right)}\right)
$$

and

$$
\left\|\mathcal{L}_{\varepsilon}^{s / 2} u_{\varepsilon}\right\|_{L^{2}\left(\mathbb{R}^{n}\right)} \leq C\left(\|f\|_{\widetilde{H}^{s}(\mathcal{O})^{*}}+\|g\|_{H^{s}\left(\mathbb{R}^{n}\right)}\right),
$$

for some constant $C>0$ independent of $f$ and $g$ and dependent on the uniform ellipticity and boundness of $A_{\varepsilon}$ and on $n$. Thus, $C$ is also independent of $\varepsilon>0$. Therefore, the sequences $\left\{u_{\varepsilon}\right\}_{\varepsilon>0}$ and $\left\{\mathcal{L}_{\varepsilon}^{s / 2} u_{\varepsilon}\right\}_{\varepsilon>0}$ remain bounded in $H^{s}\left(\mathbb{R}^{n}\right)$ (see 2.42) and $L^{2}\left(\mathbb{R}^{n}\right)$, respectively (see 2.43 ). Hence, upto a subsequence still denoted by same $\left\{u_{\varepsilon}\right\}_{\varepsilon>0}$, we get

$$
u_{\varepsilon} \rightarrow u \quad \text { weakly in } H^{s}\left(\mathbb{R}^{n}\right)
$$

and

$$
\mathcal{L}_{\varepsilon}^{s / 2} u_{\varepsilon} \rightarrow v \quad \text { weakly in } L^{2}\left(\mathbb{R}^{n}\right)
$$

In the sequel, our goal is to find the homogenized problem or the limit equation satisfied by $u \in H^{s}\left(\mathbb{R}^{n}\right)$, and also the relation between both weak limits $u$ and $v$.

To this end, we will proceed by using the extension techniques for the non-local operators, where the extended operator becomes a local operator.

\section{$3 \quad$ Extension problems for $\mathcal{L}^{s}$}

In this section, we introduce an extension problem, which characterize the non-local operator $\mathcal{L}^{s}$.

To this end, let $\mathbb{R}_{+}^{n+1}:=\left\{(x, y): x \in \mathbb{R}^{n}, y>0\right\}$ be the upper half space of $\mathbb{R}^{n+1}$ with its boundary $\partial \mathbb{R}_{+}^{n+1}:=\left\{(x, 0): x \in \mathbb{R}^{n}\right\}$. Let $\omega$ be an arbitrary $A_{2}$-Muckenhoupt weight 
function (for more details, see [14, 21]) and we denote by $L^{2}\left(\mathbb{R}_{+}^{n+1}, \omega\right)$ the weighted Sobolev space containing all functions $U$ which are defined a.e. in $\mathbb{R}_{+}^{n+1}$ such that

$$
\|U\|_{L^{2}\left(\mathbb{R}_{+}^{n+1}, \omega\right)}:=\left(\int_{\mathbb{R}_{+}^{n+1}} \omega|U|^{2} d x d y\right)^{1 / 2}<\infty .
$$

We define

$$
H^{1}\left(\mathbb{R}_{+}^{n+1}, \omega\right):=\left\{U \in L^{2}\left(\mathbb{R}_{+}^{n+1}, \omega\right): \nabla_{x, y} U \in L^{2}\left(\mathbb{R}_{+}^{n+1}, \omega\right)\right\},
$$

where $\nabla_{x, y}:=\left(\nabla, \partial_{y}\right)=\left(\nabla_{x}, \partial_{y}\right)$ is the total derivative in $\mathbb{R}_{+}^{n+1}$. In this work, the weight function $\omega$ might be $y^{1-2 s}$ (or $y^{2 s-1}$ ) and it is known that $y^{1-2 s} \in A_{2}$ for $s \in(0,1)$ (see [19]). It is easy to see that $L^{2}\left(\mathbb{R}_{+}^{n+1}, \omega\right)$ and $H^{1}\left(\mathbb{R}_{+}^{n+1}, \omega\right)$ are Banach spaces with respect to the norms $\|\cdot\|_{L^{2}\left(\mathbb{R}_{+}^{n+1}, \omega\right)}$ and

$$
\|U\|_{H^{1}\left(\mathbb{R}_{+}^{n+1}, \omega\right)}:=\left(\|U\|_{L^{2}\left(\mathbb{R}_{+}^{n+1}, \omega\right)}^{2}+\left\|\nabla_{x, y} U\right\|_{L^{2}\left(\mathbb{R}_{+}^{n+1}, \omega\right)}^{2}\right)^{1 / 2},
$$

respectively. We shall also make use of the weighted Sobolev space $H_{0}^{1}\left(\mathbb{R}_{+}^{n+1}, \omega\right)$ which is the closure of $C_{0}^{\infty}\left(\mathbb{R}_{+}^{n+1}\right)$ under the $H^{1}\left(\mathbb{R}_{+}^{n+1}, \omega\right)$-norm.

We mention that the fractional Sobolev space $H^{s}\left(\mathbb{R}^{n}\right)$ can be obtained as the trace space of the weighted Sobolev space $H^{1}\left(\mathbb{R}_{+}^{n+1}, y^{1-2 s}\right)$, for $s \in(0,1)$, (see [37]), that is,

$$
\operatorname{Tr}: H^{1}\left(\mathbb{R}_{+}^{n+1}, y^{1-2 s}\right) \rightarrow H^{s}\left(\mathbb{R}^{n}\right)
$$

is continuous. This means that, for a given $u \in H^{s}\left(\mathbb{R}^{n}\right)$, there exists $U(x, y) \in H^{1}\left(\mathbb{R}_{+}^{n+1}, y^{1-2 s}\right)$ such that $\lim _{y \rightarrow 0^{+}} U(x, y)=U(x, 0)=u(x) \in H^{s}\left(\mathbb{R}^{n}\right)$ with

$$
\|u\|_{H^{s}\left(\mathbb{R}^{n}\right)} \leq C\|U\|_{H^{1}\left(\mathbb{R}_{+}^{n+1}, y^{1-2 s}\right)} .
$$

It also follows that for any bounded open strip away from $y=0$, say

$$
D_{(a, b)}=\left\{(x, y) \in \mathbb{R}^{n} \times(a, b): 0<a<y<b<\infty\right\},
$$

we have: $U \in H^{1}\left(\mathbb{R}_{+}^{n+1}, y^{1-2 s}\right)$ for $s \in(0,1)$, implies $U \in H^{1}\left(D_{(a, b)}\right)$ and also

$$
\|U\|_{H^{1}\left(D_{(a, b)}\right)} \leq C_{a, b}\|U\|_{H^{1}\left(\mathbb{R}_{+}^{n+1}, y^{1-2 s}\right)} .
$$

This is simply a consequence of definition (3.46), since the weight $y^{1-2 s}$ is smooth enough and positive in $\overline{D_{(a, b)}}$.

Let us now consider the following extension problem in $\mathbb{R}_{+}^{n+1}$ :

$$
\begin{cases}-\mathcal{L}_{x} U+\frac{1-2 s}{y} U_{y}+U_{y y}=0 & \text { in } \mathbb{R}_{+}^{n+1}, \\ U(\cdot, 0)=u(\cdot) & \text { on } \partial \mathbb{R}_{+}^{n+1} .\end{cases}
$$

This extension problem is related to the non-local operator (2.17), where the non-local operator $\mathcal{L}^{s}$ has been regarded as a Dirichlet-to-Neumann map of the above degenerate local problem (3.50). For convenience, we construct an auxiliary matrix-valued function $\widetilde{A}: \mathbb{R}^{n} \rightarrow \mathbb{R}^{(n+1) \times(n+1)}$ by

$$
\widetilde{A}(x)=\left(\begin{array}{cc}
A(x) & 0 \\
0 & 1
\end{array}\right) .
$$


We introduce the following degenerate local operator:

$$
\mathscr{L}_{\widetilde{A}}^{1-2 s}=\nabla_{x, y} \cdot\left(y^{1-2 s} \widetilde{A}(x) \nabla_{x, y}\right) .
$$

It can be seen that $y^{-1+2 s} \mathscr{L}_{\widetilde{A}}^{1-2 s}$ is nothing else than the above degenerate local operator defined in (3.50), precisely by

$$
\mathscr{L}_{\widetilde{A}}^{1-2 s}=y^{1-2 s}\left\{\nabla \cdot(A(x) \nabla)+\frac{1-2 s}{y} \partial_{y}+\partial_{y}^{2}\right\} .
$$

Let us now recall the following existence result of the above extension problem 3.50 , which complete proof can be found in [15]:

Proposition 3.1. Let $s \in(0,1)$ and $\widetilde{A}$ be given by (3.51), with $A(x)$ satisfying the elipticity condition (1.2). Then, for given $u \in H^{s}\left(\mathbb{R}^{n}\right)$, there exists a unique minimizer of the Dirichlet functional

$$
\min _{\Psi \in H^{1}\left(\mathbb{R}_{+}^{n+1}, y^{1-2 s}\right)}\left\{\int_{\mathbb{R}_{+}^{n+1}} y^{1-2 s} \widetilde{A}(x) \nabla_{x, y} \Psi \cdot \nabla_{x, y} \Psi d x d y: \Psi(x, 0)=u(x)\right\},
$$

characterized as the unique weak solution $U \in H^{1}\left(\mathbb{R}_{+}^{n+1}, y^{1-2 s}\right)$ solving the problem

$$
\begin{cases}\mathscr{L}_{\widetilde{A}}^{1-2 s} U=0 & \text { in } \mathbb{R}_{+}^{n+1}, \\ U(\cdot, 0)=u & \text { in } \mathbb{R}^{n},\end{cases}
$$

and satisfying the following stability estimate

$$
\|U\|_{H^{1}\left(\mathbb{R}_{+}^{n+1}, y^{1-2 s}\right)} \leq C\|u\|_{H^{s}\left(\mathbb{R}^{n}\right)}
$$

for some $C>0$ independent of $u$ and $U$ and depending only on the ellipticity and boundness of $A$ and on $n$.

Proof. The proof could be find in the recent paper [15]. For our own convenience, we mention here the apriori estimate 3.55 in order to show that the constant $C>0$ appearing in 3.55 ) depends only on the ellipticity and boundness of $A$ and on $n$.

Given $u \in H^{s}\left(\mathbb{R}^{n}\right)$, there exists $U_{0}(x, y) \in H^{1}\left(\mathbb{R}_{+}^{n+1}, y^{1-2 s}\right)$ such that $\lim _{y \rightarrow 0^{+}} U_{0}(x, y)=$ $U_{0}(x, 0)=u(x)$ and by using the right continuity of the inverse trace map, we assume $\left\|U_{0}\right\|_{H^{1}\left(\mathbb{R}_{+}^{n+1}, y^{1-2 s}\right)} \leq C\|u\|_{H^{s}\left(\mathbb{R}^{n}\right)}$, where the constant $C>0$ is independent of $U_{0} \in H^{1}\left(\mathbb{R}_{+}^{n+1}, y^{1-2 s}\right)$ and $u \in H^{s}\left(\mathbb{R}^{n}\right)$.

Since $U \in H^{1}\left(\mathbb{R}_{+}^{n+1}, y^{1-2 s}\right)$ is the weak solution of $(3.54)$, let define $V:=U-U_{0}$. Then $V \in H^{1}\left(\mathbb{R}_{+}^{n+1}, y^{1-2 s}\right)$ is the weak solution of

$$
\begin{cases}\nabla \cdot\left(y^{1-2 s} \widetilde{A} \nabla V\right)=\nabla \cdot G & \text { in } \mathbb{R}_{+}^{n+1} \\ V(x, 0)=0 & \text { in } \mathbb{R}^{n}\end{cases}
$$

where $G:=-y^{1-2 s} \widetilde{A}(x) \nabla_{x, y} U_{0}$. It is easy to see that $y^{2 s-1} G \in L^{2}\left(\mathbb{R}_{+}^{n+1}, y^{1-2 s}\right)$ and

$$
\begin{aligned}
\int_{\mathbb{R}_{+}^{n+1}} y^{1-2 s}\left|y^{2 s-1} G\right|^{2} d x d y & =\int_{\mathbb{R}_{+}^{n+1}} y^{1-2 s}\left|\widetilde{A} \nabla_{x, y} U_{0}\right|^{2} d x d y \\
& \leq C \int_{\mathbb{R}_{+}^{n+1}} y^{1-2 s}\left|\nabla_{x, y} U_{0}\right|^{2} d x d y
\end{aligned}
$$


for some constant $C>0$ which depends only on boundness of $A$. Then, by multiplying (3.56) by $V \in H_{0}^{1}\left(\mathbb{R}_{+}^{n+1}, y^{1-2 s}\right)$ and integrating by parts, we get

$$
\|V\|_{H^{1}\left(\mathbb{R}_{+}^{n+1}, y^{1-2 s}\right)} \leq C\left\|y^{-1+2 s} G\right\|_{L^{2}\left(\mathbb{R}_{+}^{n+1}, y^{1-2 s}\right)},
$$

for some constant $C>0$ which depends only on the ellipticity of $A$. Finally,

$$
\begin{aligned}
\|V\|_{H^{1}\left(\mathbb{R}_{+}^{n+1}, y^{1-2 s}\right)} & \leq C\left\|U_{0}\right\|_{H^{1}\left(\mathbb{R}_{+}^{n+1}, y^{1-2 s}\right)} \leq C\|u\|_{H^{s}\left(\mathbb{R}^{n}\right)} \\
\text { or, } \quad\|U\|_{H^{1}\left(\mathbb{R}_{+}^{n+1}, y^{1-2 s}\right)} & \leq C\|u\|_{H^{s}\left(\mathbb{R}^{n}\right)},
\end{aligned}
$$

for some universal constant $C>0$ which depends only on the ellipticity and on the boundness of $A$.

As a consequence, we observe that $y^{1-2 s} \partial_{y} U$ converges to some function $h \in H^{-s}\left(\mathbb{R}^{n}\right)$, as $y \rightarrow 0$, in $H^{-s}\left(\mathbb{R}^{n}\right)$ defined as follows:

$$
(h, \phi(x, 0))_{H^{-s}\left(\mathbb{R}^{n}\right) \times H^{s}\left(\mathbb{R}^{n}\right)}=\int_{\mathbb{R}_{+}^{n+1}} y^{1-2 s} \widetilde{A}(x) \nabla_{x, y} U \cdot \nabla_{x, y} \phi d x d y,
$$

for all $\phi \in H^{1}\left(\mathbb{R}_{+}^{n+1}, y^{1-2 s}\right)$. In other words, $U \in H^{1}\left(\mathbb{R}_{+}^{n+1}, y^{1-2 s}\right)$ is the weak solution of the following Neumann boundary value problem

$$
\begin{cases}\nabla_{x, y} \cdot\left(y^{1-2 s} \widetilde{A}(x) \nabla_{x, y} U\right)=0 & \text { in } \mathbb{R}_{+}^{n+1} \\ \lim _{y \rightarrow 0^{+}} y^{1-2 s} \partial_{y} U=h & \text { in } \mathbb{R}^{n} \times\{0\} .\end{cases}
$$

The following result characterizes $\lim _{y \rightarrow 0^{+}} y^{1-2 s} \partial_{y} U=h$, as $d_{s} h=\mathcal{L}^{s} u$, for some constant $d_{s}$ depending on $s$, which connects the non-local operator $\mathcal{L}^{s}$ and the extension problem:

Proposition 3.2. Given $u \in H^{s}\left(\mathbb{R}^{n}\right)$, define

$$
U(x, y):=\int_{\mathbb{R}^{n}} P_{y}^{s}(x, z) u(z) d z
$$

where $P_{y}^{s}$ is the Poisson kernel given by

$$
P_{y}^{s}(x, z)=\frac{y^{2 s}}{4^{s} \Gamma(s)} \int_{0}^{\infty} e^{-\frac{y^{2}}{4 t}} W_{t}(x, z) \frac{d t}{t^{1+s}}, \quad x, z \in \mathbb{R}^{n}, y>0,
$$

with the heat kernel $W_{t}(x, z)$ introduced in 2.22 . Then $U \in H^{1}\left(\mathbb{R}_{+}^{n+1}, y^{1-2 s}\right)$ is the weak solution of (3.54) and

$$
\lim _{y \rightarrow 0^{+}} \frac{U(\cdot, y)-U(\cdot, 0)}{y^{2 s}}=\frac{1}{2 s} \lim _{y \rightarrow 0+} y^{1-2 s} \partial_{y} U(\cdot, y)=\frac{\Gamma(-s)}{4^{s} \Gamma(s)} \mathcal{L}^{s} u(\cdot),
$$

in $H^{-s}\left(\mathbb{R}^{n}\right)$.

Proof. The proof can be found in [34, where the authors prove the equality (3.61) for $u \in$ $\operatorname{Dom}\left(\mathcal{L}^{s}\right)$, and recently, in [15] the result has been extended for $u \in H^{s}\left(\mathbb{R}^{n}\right)$. 


\subsection{Limiting analysis of $\left\{U_{\varepsilon}\right\}_{\varepsilon>0}$ as $\varepsilon \rightarrow 0$}

We consider the following sequence of local operators:

$$
\begin{aligned}
\left\{\mathscr{L}_{\widetilde{A}_{\varepsilon}}^{1-2 s}\right\}_{\varepsilon>0} & =\left\{\nabla_{x, y} \cdot\left(y^{1-2 s} \widetilde{A}_{\varepsilon}(x) \nabla_{x, y}\right)\right\}_{\varepsilon>0} \\
& =\left\{y^{1-2 s}\left(\nabla \cdot\left(A_{\varepsilon}(x) \nabla\right)+\frac{1-2 s}{y} \partial_{y}+\partial_{y}^{2}\right)\right\}_{\varepsilon>0}
\end{aligned}
$$

introduced in (3.53), with the sequence $\left\{A_{\varepsilon}(x)\right\}_{\varepsilon>0}$ satisfying the ellipticity and boundness conditions (1.2) and regularity condition (2.11). For each $\varepsilon>0$, let us consider $U_{\varepsilon} \in H^{1}\left(\mathbb{R}_{+}^{n+1}, y^{1-2 s}\right)$ the solution of the following problem:

$$
\begin{cases}\mathscr{L}_{\widetilde{A}_{\varepsilon}}^{1-2 s} U_{\varepsilon}=0 & \text { in } \mathbb{R}_{+}^{n+1}, \\ U_{\varepsilon}(\cdot, 0)=u_{\varepsilon}(\cdot) & \text { in } \mathbb{R}^{n},\end{cases}
$$

which satisfies the stability estimate

$$
\left\|U_{\varepsilon}\right\|_{H^{1}\left(\mathbb{R}_{+}^{n+1}, y^{1-2 s}\right)} \leq C\left\|u_{\varepsilon}\right\|_{H^{s}\left(\mathbb{R}^{n}\right)} \leq C\left(\|f\|_{\widetilde{H}^{s}(\mathcal{O})^{*}}+\|g\|_{H^{s}\left(\mathbb{R}^{n}\right)}\right),
$$

for some constant $C>0$ dependent on $n$, on the uniform ellipticity and boundness of $A_{\varepsilon}$ and independent of $\varepsilon>0$. Due to the above estimate 3.63 , the sequence $\left\{U_{\varepsilon}\right\}_{\varepsilon>0}$ remains bounded in $H^{1}\left(\mathbb{R}_{+}^{n+1}, y^{1-2 s}\right)$. Therefore, upto a subsequence still denoted by same $\left\{U_{\varepsilon}\right\}_{\varepsilon>0}$, the sequence weakly converges to some limit $U \in H^{1}\left(\mathbb{R}_{+}^{n+1}, y^{1-2 s}\right)$, that is,

$$
U_{\varepsilon} \rightarrow U \quad \text { weakly in } H^{1}\left(\mathbb{R}_{+}^{n+1}, y^{1-2 s}\right) \text {. }
$$

Consequently, by the continuity of the trace map $\operatorname{Tr}: H^{1}\left(\mathbb{R}_{+}^{n+1}, y^{1-2 s}\right) \rightarrow H^{s}\left(\mathbb{R}^{n}\right)$ (see (3.48), we have

$$
\operatorname{Tr}\left(U_{\varepsilon}\right) \rightarrow \operatorname{Tr}(U) \quad \text { weakly in } H^{s}\left(\mathbb{R}^{n}\right) .
$$

Since (2.44) holds, we get that $\operatorname{Tr}\left(U_{\varepsilon}\right)=u_{\varepsilon}$ weakly converges to $u \in H^{s}\left(\mathbb{R}^{n}\right)$, hence, by the uniqueness of the weak limit in $H^{s}\left(\mathbb{R}^{n}\right)$, we find

$$
u(x)=\lim _{y \rightarrow 0^{+}} U(x, y)=U(x, 0)=\operatorname{Tr}(U) \quad \text { in } H^{s}\left(\mathbb{R}^{n}\right) .
$$

In the sequel, we look for the homogenized problem or the limit equation satisfied by $U \in H^{1}\left(\mathbb{R}_{+}^{n+1}, y^{1-2 s}\right)$. To this end, we first observe that the flux quantity $\sigma_{\varepsilon}(x, y)=$ $y^{1-2 s} \widetilde{A}_{\varepsilon}(x) \nabla_{x, y} U_{\varepsilon}(x, y)$ is uniformly bounded in $L^{2}\left(\mathbb{R}_{+}^{n+1}, y^{1-2 s}\right)^{n+1}$ because $A_{\varepsilon}(x)$ and $U_{\varepsilon} \in$ $H^{1}\left(\mathbb{R}_{+}^{n+1}, y^{1-2 s}\right)$ are uniformly bounded in their respective spaces. Thus, upto a subsequence denoted by same $\left\{\sigma_{\varepsilon}\right\}_{\varepsilon>0}$, the flux sequence has a weak limit in $L^{2}\left(\mathbb{R}_{+}^{n+1}, y^{1-2 s}\right)^{n+1}$, called it $\sigma(x, y)$, that is,

$$
\sigma_{\varepsilon}(x, y) \rightarrow \sigma(x, y) \quad \text { weakly in } L^{2}\left(\mathbb{R}_{+}^{n+1}, y^{1-2 s}\right)^{n+1} .
$$

Since $\nabla_{x, y} \cdot \sigma_{\varepsilon}(x, y)=0$ in $\mathbb{R}_{+}^{n+1}$ for all $\varepsilon>0$, and since due to 3.66 we have

$$
\nabla_{x, y} \cdot \sigma_{\varepsilon}(x, y) \rightarrow \nabla_{x, y} \cdot \sigma(x, y) \quad \text { strongly in } H^{-1}\left(\mathbb{R}_{+}^{n+1}, y^{1-2 s}\right)
$$

we find that

$$
\nabla_{x, y} \cdot \sigma(x, y)=0 \text { in } \mathbb{R}_{+}^{n+1} .
$$

Hence, our ongoing job is reduced to find the relation between $\sigma \in L^{2}\left(\mathbb{R}_{+}^{n+1}\right)^{n+1}$ and $U \in H^{1}\left(\mathbb{R}_{+}^{n+1}, y^{1-2 s}\right)$, as usual is done in the homogenization framework.

Let us now use the framework of $H$-convergence (for more details, see [23, 35]) and prove the following result: 
Lemma 3.1. Let us consider the sequence $\left\{A_{\varepsilon}\right\}_{\varepsilon}$ satisfying conditions 1.2 and that $H$ converges to $A_{*}$ (we denote by $A_{\varepsilon} \stackrel{H}{\rightarrow} A_{*}$ ) that is,

$$
A_{\varepsilon} \nabla w_{\varepsilon} \rightarrow A_{*} \nabla w \quad \text { weakly in } L^{2}\left(\mathbb{R}^{n}\right)^{n},
$$

for all test sequences $w_{\varepsilon} \in H^{1}\left(\mathbb{R}^{n}\right)$ satisfying

$$
\begin{gathered}
w_{\varepsilon} \rightarrow w \quad \text { weakly in } H^{1}\left(\mathbb{R}^{n}\right), \\
-\nabla \cdot\left(A_{\varepsilon} \nabla w_{\varepsilon}\right) \quad \text { strongly convergent in } H^{-1}\left(\mathbb{R}^{n}\right) .
\end{gathered}
$$

Then, we have

$$
y^{1-2 s} \widetilde{A}_{\varepsilon}(x) \nabla_{x, y} U_{\varepsilon}(x, y) \rightarrow y^{1-2 s} \widetilde{A}_{*}(x) \nabla_{x, y} U(x, y) \text { weakly in } L^{2}\left(\mathbb{R}_{+}^{n+1}, y^{1-2 s}\right)^{n+1},
$$

where $U \in H^{1}\left(\mathbb{R}_{+}^{n+1}, y^{1-2 s}\right)$ solves the following homogenized problem:

$$
\begin{cases}\mathscr{L}_{\widetilde{A}_{*}^{1-2 s}}^{1-2}=0 & \text { in } \mathbb{R}_{+}^{n+1} \\ U(\cdot, 0)=u(\cdot) & \text { in } \mathbb{R}^{n}\end{cases}
$$

where $u \in H^{s}\left(\mathbb{R}^{n}\right)$ and

$$
\widetilde{A}_{*}(x)=\left(\begin{array}{cc}
A_{*}(x) & 0 \\
0 & 1
\end{array}\right)
$$

Proof. Let us consider the region $D_{\left(\delta, \delta^{-1}\right)}=\left\{(x, y): x \in \mathbb{R}^{n}\right.$ and $\left.\delta<y<\delta^{-1}\right\}$, for any $\delta>0$. Since the weight $y^{1-2 s}$ is smooth enough and positive in $\overline{D_{\left(\delta, \delta^{-1}\right)}}$, then $U_{\varepsilon} \in H^{1}\left(D_{\left(\delta, \delta^{-1}\right)}\right)$ can be seen as the solution of the following uniformly elliptic equation:

$$
\nabla_{x, y} \cdot\left(y^{1-2 s} \widetilde{A}_{\varepsilon}(x) \nabla_{x, y} U_{\varepsilon}\right)=0 \quad \text { in } D_{\left(\delta, \delta^{-1}\right)} .
$$

We also get that $\left\|U_{\varepsilon}\right\|_{H^{1}\left(D_{(\delta, \delta}-1\right)}$ is uniformly bounded w.r.t. $\varepsilon$, and using 3.49) and 3.63), it follows that

$$
\left\|U_{\varepsilon}\right\|_{H^{1}\left(D_{\left(\delta, \delta^{-1}\right)}\right)} \leq C_{\delta}\left(\|f\|_{\widetilde{H}^{s}(\mathcal{O})^{*}}+\|g\|_{H^{s}\left(\mathbb{R}^{n}\right)}\right) .
$$

Thus, upto a subsequence denoted by same $U_{\varepsilon}$, the sequence weakly converges to some limit $V$ in $H^{1}\left(D_{\left(\delta, \delta^{-1}\right)}\right)$. We claim that

$$
V=\left.U\right|_{D_{(\delta, \delta-1)}},
$$

where $U \in H^{1}\left(\mathbb{R}_{+}^{n+1}, y^{1-2 s}\right)$ is the weak limit of $\left\{U_{\varepsilon}\right\}_{\varepsilon>0}$ in $H^{1}\left(\mathbb{R}_{+}^{n+1}, y^{1-2 s}\right)$ introduced in 3.64). In fact, this claim simply follows from (3.64) because

$$
\int_{D_{\left(\delta, \delta^{-1}\right)}} \varphi U_{\varepsilon} \rightarrow \int_{D_{\left(\delta, \delta^{-1}\right)}} \varphi U \quad \forall \varphi \in C_{c}^{\infty}\left(D_{\left(\delta, \delta^{-1}\right)}\right), \text { as } \varepsilon \rightarrow 0 .
$$

Let us now claim that if $A_{\varepsilon} \stackrel{H}{\rightarrow} A_{*}$, then $B_{\varepsilon}(x, y)=y^{1-2 s} \widetilde{A}_{\varepsilon}(x)$ has the following $H$-limit:

$$
B_{\varepsilon}(x, y) \stackrel{H}{\rightarrow} B_{*}(x, y)=y^{1-2 s} \widetilde{A}_{*}(x) \text { in } D_{\left(\delta, \delta^{-1}\right)} .
$$

In fact, since $U_{\varepsilon} \in H^{1}\left(D_{\left(\delta, \delta^{-1}\right)}\right)$ solves

$$
\begin{aligned}
\left(\mathcal{L}_{\varepsilon}\right)_{x} U_{\varepsilon}(x, y)=\frac{1-2 s}{y}\left(U_{\varepsilon}\right)_{y}(x, y)+\left(U_{\varepsilon}\right)_{y y} & (x, y) \\
& =y^{-1+2 s} \partial_{y}\left(y^{1-2 s} \partial_{y} U_{\varepsilon}(x, y)\right) \text { in } D_{\left(\delta, \delta^{-1}\right)}
\end{aligned}
$$


and since $U_{\varepsilon} \rightarrow U$ weakly in $H^{1}\left(D_{\left(\delta, \delta^{-1}\right)}\right)$, then we claim the right hand side of (3.71) satisfies

$$
y^{-1+2 s} \partial_{y}\left(y^{1-2 s} \partial_{y} U_{\varepsilon}(x, y)\right) \rightarrow y^{-1+2 s} \partial_{y}\left(y^{1-2 s} \partial_{y} U(x, y)\right) \text { strongly in } H^{-1}\left(D_{\left(\delta, \delta^{-1}\right.}\right) \text {. }
$$

Proof of the claim (3.72): Note that, as the strip $D_{\left(\delta, \delta^{-1}\right)}$ is bounded in $y$-direction, so by applying the standard Rellich compactness theorem (see [17]) from $U_{\varepsilon} \rightarrow U$ weakly in $H^{1}\left(D_{\left(\delta, \delta^{-1}\right)}\right)$, we get $\partial_{y} U_{\varepsilon}(x, y) \rightarrow \partial_{y} U(x, y)$ strongly in $L^{2}\left(D_{\left.\left(\delta, \delta^{-1}\right)\right)}\right.$. So, $y^{1-2 s} \partial_{y} U_{\varepsilon}(x, y) \rightarrow$ $y^{1-2 s} \partial_{y} U(x, y)$ strongly in $L^{2}\left(D_{\left.\left(\delta, \delta^{-1}\right)\right)}\right.$. Following that, we have

$$
y^{-1+2 s} \partial_{y}\left(y^{1-2 s} \partial_{y} U_{\varepsilon}(x, y)\right) \rightarrow y^{-1+2 s} \partial_{y}\left(y^{1-2 s} \partial_{y} U_{\varepsilon}(x, y)\right)
$$

weakly in $L^{2}\left(D_{\left(\delta, \delta^{-1}\right)}\right)$, therefore in $H^{-1}$ strong topology; that is, for any $\phi(x, y) \in C_{c}^{\infty}\left(D_{\left(\delta, \delta^{-1}\right)}\right)$, we have

$$
\begin{aligned}
\int_{D_{\left(\delta, \delta^{-1}\right)}} y^{-1+2 s} \partial_{y} & \left(y^{1-2 s} \partial_{y} U_{\varepsilon}(x, y)\right) \phi(x, y) d x d y \\
= & \int_{D_{\left(\delta, \delta^{-1}\right)}}\left(y^{1-2 s} \partial_{y} U_{\varepsilon}(x, y)\right) \partial_{y}\left(y^{-1+2 s} \phi(x, y)\right) d x d y \\
& \rightarrow \int_{D_{\left(\delta, \delta^{-1}\right)}}\left(y^{1-2 s} \partial_{y} U(x, y)\right) \partial_{y}\left(y^{-1+2 s} \phi(x, y)\right) d x d y \\
& =\int_{D_{\left(\delta, \delta^{-1}\right)}} y^{-1+2 s} \partial_{y}\left(y^{1-2 s} \partial_{y} U(x, y)\right) \phi(x, y) d x d y
\end{aligned}
$$

This establishes our above claim (3.72).

Thus, by passing to the limit in (3.71), as $\varepsilon \rightarrow 0$, we obtain the following homogenized equation:

$$
\left(\mathcal{L}_{*}\right)_{x} U(x, y)=\frac{1-2 s}{y} U_{y}(x, y)+U_{y y}(x, y) \quad \text { in } D_{\left(\delta, \delta^{-1}\right)},
$$

where $\left(\mathcal{L}_{*}\right)_{x}=-\nabla_{x} \cdot\left(A_{*}(x) \nabla_{x}\right)$. Moreover, we get the flux convergence

$$
y^{1-2 s} \widetilde{A}_{\varepsilon}(x) \nabla_{x, y} U_{\varepsilon}(x, y) \rightarrow y^{1-2 s} \widetilde{A}_{*}(x) \nabla_{x, y} U(x, y) \text { weakly in } L^{2}\left(D_{\left(\delta, \delta^{-1}\right)}\right)^{n+1} \text {. }
$$

Thus, $U \in H^{1}\left(D_{\left(\delta, \delta^{-1}\right)}\right)$ solves

$$
\nabla_{x, y} \cdot\left(y^{1-2 s} \widetilde{A}_{*}(x) \nabla_{x, y} U(x, y)\right)=0 \text { in } D_{\left(\delta, \delta^{-1}\right)},
$$

which concludes 3.70 .

Since $(3.70)$ holds for any $\delta>0$ small enough, and $y^{1-2 s} \widetilde{A}_{*}(x) \nabla_{x, y} U(x, y) \in L^{2}\left(\mathbb{R}_{+}^{n+1}, y^{1-2 s}\right)^{n+1}$, we would like to claim that $U \in H^{1}\left(\mathbb{R}_{+}^{n+1}, y^{1-2 s}\right)$ is solution of

$$
\nabla_{x, y} \cdot\left(y^{1-2 s} \widetilde{A}_{*}(x) \nabla_{x, y} U\right)=0 \quad \text { in } \mathbb{R}_{+}^{n+1}=\cup_{\delta>0} D_{\left(\delta, \delta^{-1}\right)}
$$

and we have the flux convergence $(3.67)$ in $L^{2}\left(\mathbb{R}_{+}^{n+1}, y^{1-2 s}\right)^{n+1}$. In order to justify our claim 3.73 , we need to show $U \in H^{1}\left(\mathbb{R}_{+}^{n+1}, y^{1-2 s}\right)$ satisfy

$$
\int_{\mathbb{R}_{+}^{n+1}}\left(y^{1-2 s} \widetilde{A}_{*}(x) \nabla_{x, y} U(x, y)\right) \cdot \nabla_{x, y} \varphi(x, y) d x d y=0 \quad \text { for all } \varphi \in C_{c}^{\infty}\left(\mathbb{R}_{+}^{n+1}\right) .
$$

Since $\varphi \in C_{c}^{\infty}\left(\mathbb{R}_{+}^{n+1}\right)$ implies there exists $\delta>0$ such that $\varphi \in C_{c}^{\infty}\left(D_{\left(\delta, \delta^{-1}\right)}\right)$, and then, from (3.70), we have

$$
\begin{aligned}
\int_{\mathbb{R}_{+}^{n+1}}\left(y^{1-2 s} \widetilde{A}_{*}(x) \nabla_{x, y} U(x, y)\right) & \cdot \nabla_{x, y} \varphi(x, y) d x d y \\
= & \int_{\left.D_{(\delta, \delta}-1\right)}\left(y^{1-2 s} \widetilde{A}_{*}(x) \nabla_{x, y} U(x, y)\right) \cdot \nabla_{x, y} \varphi(x, y) d x d y=0 .
\end{aligned}
$$


Since $\sigma(x, y)$ is the weak limit of $\sigma_{\varepsilon}(x, y)$ in $L^{2}\left(\mathbb{R}_{+}^{n+1}, y^{1-2 s}\right)^{n+1}$ (see 3.66$)$ ), and

$$
y^{1-2 s} \widetilde{A}_{*}(x) \nabla_{x, y} U(x, y) \in L^{2}\left(\mathbb{R}_{+}^{n+1}, y^{1-2 s}\right)^{n+1},
$$

then

$$
\sigma(x, y)=y^{1-2 s} \widetilde{A}_{*}(x) \nabla_{x, y} U(x, y) \text { in } \mathbb{R}_{+}^{n+1},
$$

that is, we want to show that

$$
\begin{aligned}
& \int_{\mathbb{R}_{+}^{n+1}}\left(y^{1-2 s} \widetilde{A}_{\varepsilon}(x) \nabla_{x, y} U_{\varepsilon}(x, y)\right) \varphi(x, y) d x d y \\
& \rightarrow \int_{\mathbb{R}_{+}^{n+1}}\left(y^{1-2 s} \widetilde{A}_{*}(x) \nabla_{x, y} U(x, y)\right) \varphi d x d y \quad \text { for all } \varphi \in C_{c}^{\infty}\left(\mathbb{R}_{+}^{n+1}\right) .
\end{aligned}
$$

Let us show our claim (3.74) in a similar way. Since $\varphi \in C_{c}^{\infty}\left(\mathbb{R}_{+}^{n+1}\right)$ implies there exists $\delta>0$ such that $\varphi \in C_{c}^{\infty}\left(D_{\left(\delta, \delta^{-1}\right)}\right)$, so from $\sigma(x, y)$ is the weak limit of $\sigma_{\varepsilon}(x, y)$ in $L^{2}\left(D_{\left(\delta, \delta^{-1}\right)}\right)$, we have the desired conclusion (3.74).

Then combining with the fact $U(x, 0)=u(x) \in H^{s}\left(\mathbb{R}^{n}\right)$ due to (3.65), we establish that the homogenized boundary value problem $(3.68)$ is satisfied by $U \in H^{1}\left(\mathbb{R}_{+}^{n+1}, y^{1-2 s}\right)$.

\section{Proof of Theorem 1.1}

We first use Proposition 3.2 and we get

$$
\lim _{y \rightarrow 0^{+}} \frac{U_{\varepsilon}(\cdot, y)-U_{\varepsilon}(\cdot, 0)}{y^{2 s}}=\frac{1}{2 s} \lim _{y \rightarrow 0^{+}} y^{1-2 s} \partial_{y} U_{\varepsilon}(\cdot, y)=\frac{\Gamma(-s)}{4^{s} \Gamma(s)} \mathcal{L}_{\varepsilon}^{s} u_{\varepsilon} \quad \text { in } H^{-s}\left(\mathbb{R}^{n}\right),
$$

where $U_{\varepsilon} \in H^{1}\left(\mathbb{R}_{+}^{n+1}, y^{1-2 s}\right)$ is the weak solution of problem 3.62$)$, and we also have

$$
\lim _{y \rightarrow 0^{+}} \frac{U(\cdot, y)-U(\cdot, 0)}{y^{2 s}}=\frac{1}{2 s} \lim _{y \rightarrow 0^{+}} y^{1-2 s} \partial_{y} U(\cdot, y)=\frac{\Gamma(-s)}{4^{s} \Gamma(s)} \mathcal{L}_{*}^{s} u \quad \text { in } H^{-s}\left(\mathbb{R}^{n}\right),
$$

where $U \in H^{1}\left(\mathbb{R}_{+}^{n+1}, y^{1-2 s}\right)$ is the weak solution of the homogenized problem (3.68).

Next, due to (3.57) and (3.58), we have

$$
\left(\lim _{y \rightarrow 0^{+}} y^{1-2 s} \partial_{y} U_{\varepsilon}(x, y), \phi(x, 0)\right)_{H^{-s}\left(\mathbb{R}^{n}\right) \times H^{s}\left(\mathbb{R}^{n}\right)}
$$

$$
=\int_{\mathbb{R}_{+}^{n+1}} y^{1-2 s} \widetilde{A}_{\varepsilon}(x) \nabla_{x, y} U_{\varepsilon} \cdot \nabla_{x, y} \phi d x d y
$$

and

$$
\left(\lim _{y \rightarrow 0^{+}} y^{1-2 s} \partial_{y} U(x, y), \phi(x, 0)\right)_{H^{-s}\left(\mathbb{R}^{n}\right) \times H^{s}\left(\mathbb{R}^{n}\right)}
$$

$$
=\int_{\mathbb{R}_{+}^{n+1}} y^{1-2 s} \widetilde{A}(x) \nabla_{x, y} U \cdot \nabla_{x, y} \phi d x d y
$$

for all $\phi \in H^{1}\left(\mathbb{R}_{+}^{n+1}, y^{1-2 s}\right)$. 
Let us now pass to the limit in the above identity, as $\varepsilon \rightarrow 0$, and use the flux convergence (3.67) to find that

$$
\begin{aligned}
\left(\lim _{y \rightarrow 0^{+}} y^{1-2 s} \partial_{y} U_{\varepsilon}(x, y), \phi(x, 0)\right)_{H^{-s}\left(\mathbb{R}^{n}\right) \times H^{s}\left(\mathbb{R}^{n}\right)} & \rightarrow\left(\lim _{y \rightarrow 0^{+}} y^{1-2 s} \partial_{y} U(x, y), \phi(x, 0)\right)_{H^{-s}\left(\mathbb{R}^{n}\right) \times H^{s}\left(\mathbb{R}^{n}\right)},
\end{aligned}
$$

for all $\phi \in H^{1}\left(\mathbb{R}_{+}^{n+1}, y^{1-2 s}\right)$.

Then, by taking $\phi(x, 0)=\psi(x) \in C_{c}^{\infty}\left(\mathbb{R}^{n}\right)$ (which is clearly possible), and using (4.75), 4.76 and 4.77, we obtain

$$
\left(\mathcal{L}_{\varepsilon}^{s} u_{\varepsilon}(x), \psi(x)\right)_{H^{-s}\left(\mathbb{R}^{n}\right) \times H^{s}\left(\mathbb{R}^{n}\right)} \rightarrow\left(\mathcal{L}_{*}^{s} u(x), \psi(x)\right)_{H^{-s}\left(\mathbb{R}^{n}\right) \times H^{s}\left(\mathbb{R}^{n}\right)} \text { as } \varepsilon \rightarrow 0,
$$

for all $\psi \in C_{c}^{\infty}\left(\mathbb{R}^{n}\right)$.

We now choose $\operatorname{Supp} \psi \subset \mathcal{O}$, and since $\mathcal{L}_{\varepsilon}^{s} u_{\varepsilon}=f$ in $\mathcal{O}$ due to 2.41), we therefore obtain

$$
\mathcal{L}_{*}^{s} u=f \quad \text { in } \mathcal{O} .
$$

On the other hand, since $u_{\varepsilon}=g$ in $\mathcal{O}_{e}$, then as a $H^{s}\left(\mathbb{R}^{n}\right)$-weak limit of the sequence $\left\{u_{\varepsilon}\right\}_{\varepsilon>0}$, we get

$$
u=g \quad \text { in } \mathcal{O}_{e} .
$$

We thus obtained the homogenized equation: $u \in H^{s}\left(\mathbb{R}^{n}\right)$ is the unique solution of the following non-local problem:

$$
\begin{cases}\mathcal{L}_{*}^{s} u=\left(-\nabla \cdot\left(A_{*}(x) \nabla\right)^{s} u=f\right. & \text { in } \mathcal{O} \\ u=g & \text { in } \mathcal{O}_{e}\end{cases}
$$

for $f \in \widetilde{H}^{s}(\mathcal{O})^{*}$ and $g \in H^{s}\left(\mathbb{R}^{n}\right)$.

Let us now prove the energy convergence, precisely

$$
\left\|\mathcal{L}_{\varepsilon}^{s / 2} u_{\varepsilon}\right\|_{L^{2}\left(\mathbb{R}^{n}\right)} \rightarrow\left\|\mathcal{L}_{*}^{s / 2} u\right\|_{L^{2}\left(\mathbb{R}^{n}\right)}, \quad \text { as } \varepsilon \rightarrow 0 .
$$

To this end, we multiply 3.62 by $U_{\varepsilon} \in H^{1}\left(\mathbb{R}_{+}^{n+1}, y^{1-2 s}\right)$, we integrate by parts, and we pass to the limit as $\varepsilon \rightarrow 0$, obtaining

$$
\int_{\mathbb{R}_{+}^{n+1}} y^{1-2 s} \widetilde{A}_{\varepsilon}(x) \nabla_{x, y} U_{\varepsilon} \cdot \nabla_{x, y} U_{\varepsilon} d x d y \rightarrow \int_{\mathbb{R}_{+}^{n+1}} y^{1-2 s} \widetilde{A}_{*}(x) \nabla_{x, y} U \cdot \nabla_{x, y} U d x d y .
$$

Consequently, the convergence 4.79 holds.

Finally, we are left out to show the flux convergence $(1.3)$. To this end, let us observe that our analysis simply suggests that, if we take $\frac{s}{2}$ instead of $s \in(0,1)$ in $(3.62)$, which is clearly possible and independent of the main problem (2.41) to consider, then it follows from (4.78) that

$$
\mathcal{L}_{\varepsilon}^{s / 2} u_{\varepsilon} \rightarrow \mathcal{L}_{*}^{s / 2} u \quad \text { weakly in } H^{-s / 2}\left(\mathbb{R}^{n}\right)
$$

Since $\left\{\mathcal{L}_{\varepsilon}^{s / 2} u_{\varepsilon}\right\}_{\varepsilon>0} \subseteq L^{2}\left(\mathbb{R}^{n}\right)$ has a $L^{2}\left(\mathbb{R}^{n}\right)$ weak sub-sequential limit $v \in L^{2}\left(\mathbb{R}^{n}\right)$ (see 2.45) and $\mathcal{L}_{*}^{s / 2} u \in L^{2}\left(\mathbb{R}^{n}\right)$, thus $v=\mathcal{L}_{*}^{s / 2} u$. Therefore,

$$
\mathcal{L}_{\varepsilon}^{s / 2} u_{\varepsilon} \rightarrow \mathcal{L}_{*}^{s / 2} u \quad \text { weakly in } L^{2}\left(\mathbb{R}^{n}\right) .
$$

This completes the proof of Theorem 1.1 . 


\section{Non-local homogenization in perforated domain}

Let us consider the sequence of closed subsets $\left\{T_{\varepsilon}\right\}_{\varepsilon>0}$ which are called holes and the perforated domain $\mathcal{O}_{\varepsilon}$ defined in $(1.5)$ with the condition on the Lebesgue measure (1.6).

For $s \in(0,1)$ and for $\varepsilon>0$, we consider the following non-local Dirichlet problem associated with the fractional Laplace operator described in (1.7), precisely:

$$
\begin{cases}(-\Delta)^{s} u_{\varepsilon}=f & \text { in } \mathcal{O}_{\varepsilon}, \\ u_{\varepsilon}=g & \text { in } \mathbb{R}^{n} \backslash \mathcal{O}_{\varepsilon},\end{cases}
$$

for some $f \in \widetilde{H}^{s}(\mathcal{O})^{*}$ and $g \in H^{s}\left(\mathbb{R}^{n}\right)$. We recall from 2.29 that, for $v \in H^{s}\left(\mathbb{R}^{n}\right)$,

$$
(-\Delta)^{s} v(x)=c_{n, s} \mathrm{P} . \mathrm{V} \cdot \int_{\mathbb{R}^{n}} \frac{v(x)-v(y)}{|x-y|^{n+2 s}} d y,
$$

with $c_{n, s}=\frac{\Gamma\left(\frac{n}{2}+s\right)}{|\Gamma(-s)|} \frac{4^{s}}{\pi^{n / 2}}$, and P.V. stands for the standard principal value operator. Then, we define the bilinear form as: for any $v, w \in H^{s}\left(\mathbb{R}^{n}\right)$,

$$
\begin{aligned}
\mathcal{B}^{s}(v, w): & =c_{n, s} \int_{\mathbb{R}^{n}} \int_{\mathbb{R}^{n}} \frac{(v(x)-v(z))(w(x)-w(z))}{|x-z|^{n+2 s}} d x d z \\
& =\int_{\mathbb{R}^{n}}(-\Delta)^{s / 2} v(-\Delta)^{s / 2} w d x .
\end{aligned}
$$

Then for each fixed $\varepsilon>0$, there exists a unique solution $u_{\varepsilon} \in H^{s}\left(\mathbb{R}^{n}\right)$ such that

$$
\mathcal{B}^{s}\left(u_{\varepsilon}, w\right)=\langle f, w\rangle \text { for any } w \in \widetilde{H}^{s}\left(\mathcal{O}_{\varepsilon}\right) \text { with } u_{\varepsilon}-g \in \widetilde{H}^{s}\left(\mathcal{O}_{\varepsilon}\right),
$$

for any $f \in \widetilde{H}^{s}(\mathcal{O})^{*}$ and $g \in H^{s}\left(\mathbb{R}^{n}\right)$, where $\langle\cdot, \cdot\rangle$ stands for the duality pairing between $\left(\widetilde{H}^{s}\right)^{*}$ and $\widetilde{H}^{s}$. The above existence and uniqueness result is a direct consequence of Proposition 2.1 .

Let us first note that $u_{\varepsilon} \in H^{s}\left(\mathbb{R}^{n}\right)$ is already defined everywhere in the entire space. We now consider the bilinear form (5.82) with $w=u_{\varepsilon}-g \in H^{s}\left(\mathbb{R}^{n}\right)$ and use definition (5.81) in order to get

$$
\int_{\mathbb{R}^{n}}(-\Delta)^{s / 2} u_{\varepsilon}(-\Delta)^{s / 2} u_{\varepsilon} d x-\int_{\mathbb{R}^{n}}(-\Delta)^{s / 2} u_{\varepsilon}(-\Delta)^{s / 2} g d x=\left\langle f, u_{\varepsilon}-g\right\rangle,
$$

or

$$
\frac{1}{2}\left\|(-\Delta)^{s / 2} u_{\varepsilon}\right\|_{L^{2}\left(\mathbb{R}^{n}\right)}^{2} \leq \frac{1}{2}\left\|(-\Delta)^{s / 2} g\right\|_{L^{2}\left(\mathbb{R}^{n}\right)}^{2}+\|f\|_{\widetilde{H}^{s}(\mathcal{O})^{*}}\left\|u_{\varepsilon}-g\right\|_{H^{s}\left(\mathbb{R}^{n}\right)} .
$$

Since $\mathcal{O}$ is bounded, $u_{\varepsilon}-g=0$ in $\mathcal{O}_{e}$, and the Hardy-Littlewood-Sobolev inequality (see [33]) holds, we obtain

$$
\left\|u_{\varepsilon}-g\right\|_{L^{2}\left(\mathbb{R}^{n}\right)} \leq C_{\mathcal{O}}\left\|u_{\varepsilon}-g\right\|_{L^{\frac{2 n}{n-2 s}\left(\mathbb{R}^{n}\right)}} \leq C\left\|(-\Delta)^{s / 2}\left(u_{\varepsilon}-g\right)\right\|_{L^{2}\left(\mathbb{R}^{n}\right)},
$$

then, due to estimate 5.83 we deduce that

$$
\left\|u_{\varepsilon}\right\|_{H^{s}\left(\mathbb{R}^{n}\right)} \leq C\left(\|f\|_{\widetilde{H}^{s}(\mathcal{O})^{*}}+\|g\|_{H^{s}\left(\mathbb{R}^{n}\right)}\right),
$$

where the constant $C$ is independent of $\varepsilon>0$. Consequently, upto a subsequence, still denoted by $\left\{u_{\varepsilon}\right\}_{\varepsilon>0}$, we have

$$
u_{\varepsilon} \rightarrow u \quad \text { weakly in } H^{s}\left(\mathbb{R}^{n}\right),
$$

for some $u \in H^{s}\left(\mathbb{R}^{n}\right)$.

Our goal is to find the problem satisfied by the limit $u \in H^{s}\left(\mathbb{R}^{n}\right)$, precisely the homogenized problem. To accomplish this we recall the standard homogenization framework for the Laplacian operator in perforated domain, following the work of Cioranescu and Murat in [10, Chapter 4]. 


\section{Homogenization framework in perforated domain}

Let us assume that there exist a sequence of functions $\left\{w_{\varepsilon}\right\}_{\varepsilon>0}$ such that satisfies the following three hypotheses:

(H1) $w_{\varepsilon} \in H^{1}(\mathcal{O})$;

(H2) $w_{\varepsilon}=0$ on the holes $\underset{0<\delta \leq \varepsilon}{\cup} T_{\delta}$;

(H3) $w_{\varepsilon} \rightarrow 1$ weakly in $H^{1}(\mathcal{O})$.

Such sequences exist and can be constructed for the inhomogeneities governed by spherical, elliptical, cylindrical holes, etc., in dimension $n \geq 2$. Let us recall here one example of such sequences (for more details, see [10, Chapter 4]):

Example 5.1 (Spherical holes periodically distributed in volume). For each value of $\varepsilon>0$, one covers $\mathbb{R}^{n}(n \geq 2)$ by cubes $Y_{\varepsilon}$ of size $2 \varepsilon$. From each cube we remove the ball $T_{\varepsilon}$ of radius $a_{\varepsilon}>0$ and both, cube and ball, share the same center. In this way, $\mathbb{R}^{n}$ is perforated by spherical identical holes as

$$
\mathcal{O}_{\varepsilon}=\mathcal{O} \cap\left(\mathbb{R}^{n} \backslash \underset{0<\delta \leq \varepsilon}{\cup} T_{\delta}\right)
$$

which means that we remove from $\mathcal{O}$ small balls of radius $a_{\varepsilon}$, whose centers are the nodes of a lattice in $\mathbb{R}^{n}$ with cell size $2 \varepsilon$.
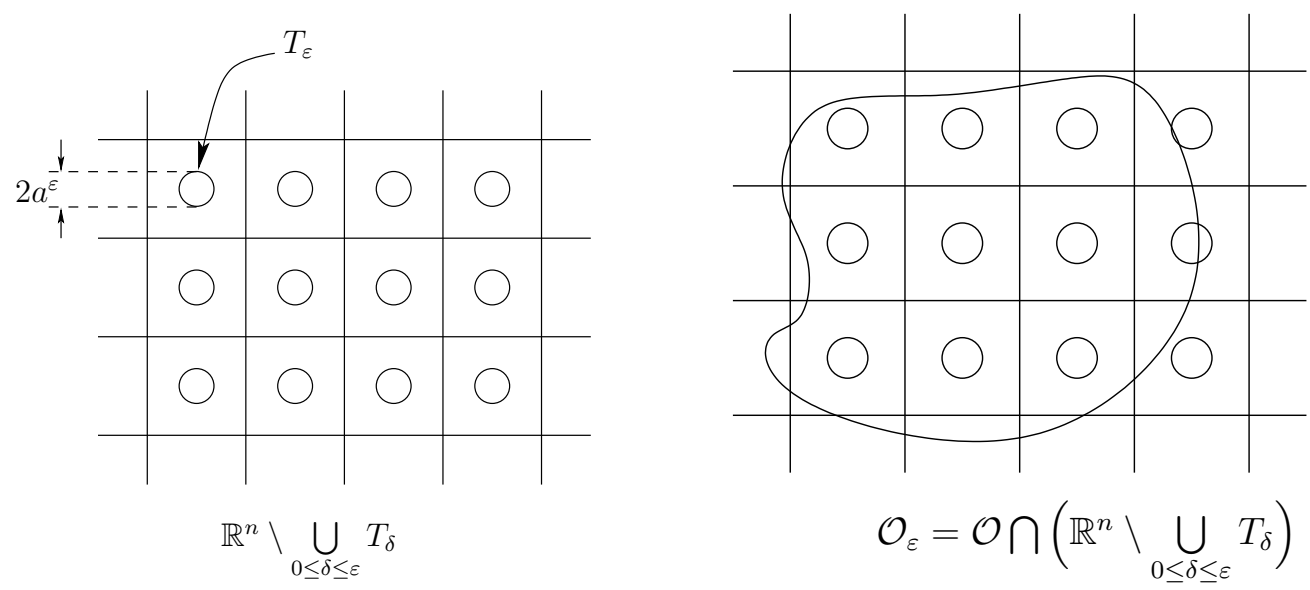

Figure 1: Spherical holes periodically distributed in volume.

In this case, one constructs $w_{\varepsilon}$ in polar coordinates in the annulus $B_{\varepsilon} \backslash T_{\varepsilon}$ as follows:

$$
w_{\varepsilon}(r)= \begin{cases}\frac{\ln a_{\varepsilon}-\ln r}{\ln a_{\varepsilon}-\ln \varepsilon} & \text { if } n=2, \\ \frac{a_{\varepsilon}^{-(n-2)}-r^{-(n-2)}}{a_{\varepsilon}^{-(n-2)}-\varepsilon^{-(n-2)}} & \text { if } n \geq 3,\end{cases}
$$

where $r=|x|$.

\section{Proof of Theorem 1.2}

By hypotheses (H1) and (H2), for any $\varphi \in \mathcal{D}(\mathcal{O})$, the sequence $\left\{w_{\varepsilon} \varphi\right\}_{\varepsilon>0} \subseteq \widetilde{H}^{s}(\mathcal{O})$ with $S u p p w_{\varepsilon} \varphi \subset \overline{\mathcal{O}}$ (or lies in $H_{\overline{\mathcal{O}}}^{s}\left(\mathbb{R}^{n}\right)$ ). Thus, one can take $w_{\varepsilon} \varphi \in \widetilde{H}^{s}(\mathcal{O})$ as test function in the 
variational formulation 5.82 to obtain

$$
\int_{\mathbb{R}^{n}}(-\Delta)^{s / 2} u_{\varepsilon}(-\Delta)^{s / 2}\left(w_{\varepsilon} \varphi\right) d x=\left\langle f, w_{\varepsilon} \varphi\right\rangle_{\widetilde{H}^{s}(\mathcal{O})^{*}, \widetilde{H}^{s}(\mathcal{O})} .
$$

Since $u_{\varepsilon}$ weakly converges to $u$ in $H^{s}\left(\mathbb{R}^{n}\right)$ due to 5.84 , then

$$
(-\Delta)^{s / 2} u_{\varepsilon} \rightarrow(-\Delta)^{s / 2} u \quad \text { weakly in } L^{2}\left(\mathbb{R}^{n}\right) .
$$

Next, due to hypothesis (H3), we have

$$
w_{\varepsilon} \varphi \rightarrow \varphi \quad \text { weakly in } H_{\overline{\mathcal{O}}}^{1}\left(\mathbb{R}^{n}\right) .
$$

At this point, we recall the Rellich Theorem from [17, Theorem 8.2, pp.199], which says that: for $t_{1}<t_{2}$ with $t_{1}, t_{2} \in \mathbb{R}$, the inclusion map

$$
H_{\overline{\mathcal{O}}}^{t_{2}}\left(\mathbb{R}^{n}\right) \hookrightarrow H^{t_{1}}\left(\mathbb{R}^{n}\right) \text { is compact, }
$$

then choosing $t_{1}=s \in(0,1)$ and $t_{2}=1$, 5.87) gives us the following strong convergence:

$$
w_{\varepsilon} \varphi \rightarrow \varphi \quad \text { strongly in } H^{s}\left(\mathbb{R}^{n}\right) \text {, as } \varepsilon \rightarrow 0 .
$$

Thus, we deduce

$$
(-\Delta)^{s / 2} w_{\varepsilon} \varphi \rightarrow(-\Delta)^{s / 2} \varphi \quad \text { strongly in } L^{2}\left(\mathbb{R}^{n}\right) .
$$

We can now pass to the limit in identity (5.85) and by using the weak convergence (5.86) and the strong convergence (5.88), we obtain

$$
\int_{\mathbb{R}^{n}}(-\Delta)^{s / 2} u(-\Delta)^{s / 2} \varphi d x=\langle f, \varphi\rangle_{\widetilde{H}^{s}(\mathcal{O})^{*}, \widetilde{H}^{s}(\mathcal{O})},
$$

which can be written as follows:

$$
\mathcal{B}^{s}(u, \varphi)=\langle f, \varphi\rangle \text { for any } \varphi \in \widetilde{H}^{s}(\mathcal{O}) \text { with } u-g \in \widetilde{H}^{s}(\mathcal{O}) .
$$

Hence, $u \in H^{s}\left(\mathbb{R}^{n}\right)$ uniquely solves the homogenized equation

$$
\begin{cases}(-\Delta)^{s} u=f & \text { in } \mathcal{O}, \\ u=g & \text { in } \mathbb{R}^{n} \backslash \mathcal{O},\end{cases}
$$

for $f \in \widetilde{H}^{s}(\mathcal{O})^{*}$ and $g \in H^{s}\left(\mathbb{R}^{n}\right)$. This completes the proof of Theorem 1.2 .

Remark 5.1. Let us observe that the problem associated with the local operator $-\Delta$, precisely

$$
-\Delta u_{\varepsilon}=f \quad \text { in } \mathcal{O}_{\varepsilon}, \quad u_{\varepsilon} \in H_{0}^{1}\left(\mathcal{O}_{\varepsilon}\right),
$$

with $f \in L^{2}(\mathcal{O})$, the $H_{0}^{1}(\mathcal{O})$-weak limit, as $\varepsilon \rightarrow 0$, of the extension sequence $\left\{\widetilde{u}_{\varepsilon}=\chi_{\mathcal{O}_{\varepsilon}} u_{\varepsilon}\right\}_{\varepsilon>0} \subseteq$ $H_{0}^{1}(\mathcal{O})$, say $u \in H_{0}^{1}(\mathcal{O})$, solves the following homogenized problem:

$$
-\Delta u+\mu u=f \quad \text { in } \mathcal{O}, \quad u \in H_{0}^{1}(\mathcal{O}),
$$

where the so-called "strange term" $\mu \in W^{-1, \infty}(\mathcal{O})$, defined along the hypotheses (H1), (H2), (H3) and the following convergence: for every sequence $v_{\varepsilon}$ such that $v_{\varepsilon}=0$ on $T_{\varepsilon}$ satisfying $v_{\varepsilon} \rightarrow v$ weakly in $H^{1}(\mathcal{O})$ (with $v \in H^{1}(\mathcal{O})$ ), one has

$$
\left\langle-\Delta w_{\varepsilon}, \varphi v_{\varepsilon}\right\rangle_{\left(H^{-1}(\mathcal{O}), H_{0}^{1}(\mathcal{O})\right)} \rightarrow\langle\mu, \varphi v\rangle_{\left(H^{-1}(\mathcal{O}), H_{0}^{1}(\mathcal{O})\right)}, \quad \text { for all } \varphi \in \mathcal{D}(\mathcal{O}) .
$$


Nevertheless, in our non-local problem we do not find any additional term as $\mu u$. The key difference is that, under the same hypotheses on $\left\{w_{\varepsilon}\right\}_{\varepsilon>0}$, the strong convergence result (5.88) fails whenever $s=1$. Since $(-\Delta)^{s} w \rightarrow(-\Delta) w$ in $L^{2}(\mathcal{O})$ for $w \in H^{2}(\mathcal{O})$ as $s \rightarrow 1^{-}$(see [11]), so this tells us the homogenization process as $\varepsilon \rightarrow 0$ in perforated domain might not stable under $s \rightarrow 1^{-}$unless $\mu \equiv 0$. In our previous Example 5.1. with $T_{\varepsilon}$ as a periodic network of balls of radius $a_{\varepsilon}$ and centered in $2 \pi \varepsilon \mathbb{Z}^{N}, \mu$ becomes 0 only if (see [12, Theorem 2.1]):

$$
\lim _{\varepsilon \rightarrow 0} \frac{-\left(\ln a_{\varepsilon}\right)^{-1}}{\varepsilon^{2}}=0, \text { for } n=2, \quad \lim _{\varepsilon \rightarrow 0} \frac{a_{\varepsilon}}{\varepsilon^{3}}=0, \text { for } n \geq 3 .
$$

Then, in this case, we can say the limiting process as $\varepsilon \rightarrow 0$ and $s \rightarrow 1^{-}$are interchangeable.

\section{References}

[1] Grégoire Allaire. Shape optimization by the homogenization method, volume 146 of Applied Mathematical Sciences. Springer-Verlag, New York, 2002.

[2] David Applebaum. Lévy processes and stochastic calculus, volume 116 of Cambridge Studies in Advanced Mathematics. Cambridge University Press, Cambridge, second edition, 2009.

[3] Mariko Arisawa. Homogenization of a class of integro-differential equations with Lévy operators. Comm. Partial Differential Equations, 34(7-9):617-624, 2009.

[4] D. G. Aronson. Bounds for the fundamental solution of a parabolic equation. Bull. Amer. Math. Soc., 73:890-896, 1967.

[5] Alain Bensoussan, Jacques-Louis Lions, and George Papanicolaou. Asymptotic analysis for periodic structures, volume 5 of Studies in Mathematics and its Applications. NorthHolland Publishing Co., Amsterdam-New York, 1978.

[6] Jean Bertoin. Lévy processes, volume 121 of Cambridge Tracts in Mathematics. Cambridge University Press, Cambridge, 1996.

[7] Claudia Bucur and Enrico Valdinoci. Nonlocal diffusion and applications, volume 20 of Lecture Notes of the Unione Matematica Italiana. Springer, [Cham]; Unione Matematica Italiana, Bologna, 2016.

[8] Luis Caffarelli and Luis Silvestre. An extension problem related to the fractional Laplacian. Communications in partial differential equations, 32(8):1245-1260, 2007.

[9] Luis A Caffarelli and Pablo Raúl Stinga. Fractional elliptic equations, Caccioppoli estimates and regularity. Annales de l'Institut Henri Poincare (C) Non Linear Analysis, 33(3):767-807, 2016.

[10] Andrej Cherkaev and Robert Kohn, editors. Topics in the mathematical modelling of composite materials. Progress in Nonlinear Differential Equations and their Applications, 31. Birkhäuser Boston, Inc., Boston, MA, 1997.

[11] Eleonora Di Nezza, Giampiero Palatucci, and Enrico Valdinoci. Hitchhiker's guide to the fractional Sobolev spaces. Bull. Sci. Math., 136(5):521-573, 2012.

[12] Delphine Dupuy, Rafael Orive, and Loredana Smaranda. Bloch waves homogenization of a Dirichlet problem in a periodically perforated domain. Asymptot. Anal., 61(3-4):229$250,2009$.

[13] Stewart N. Ethier and Thomas G. Kurtz. Markov processes. Wiley Series in Probability and Mathematical Statistics: Probability and Mathematical Statistics. John Wiley \& Sons, Inc., New York, 1986. Characterization and convergence. 
[14] Eugene B Fabes, Carlos Kenig, and Raul P Serapioni. The local regularity of solutions of degenerate elliptic equations. Communications in Statistics-Theory and Methods, $7(1): 77-116,1982$.

[15] Tuhin Ghosh, Yi-Hsuan Lin, and Xiao Jingni. The Calderón problem for variable coefficients nonlocal elliptic operators. Communications in Partial Differential Equations, 42(12):1923-1961, 2017.

[16] Alexander Grigor'yan. Heat kernel and analysis on manifolds, volume 47. American Mathematical Society Providence, 2009.

[17] Gerd Grubb. Distributions and Operators. Springer-Verlag, New York, 2008.

[18] V. V. Jikov, S. M. Kozlov, and O. A. Oleı̆nik. Homogenization of differential operators and integral functionals. Springer-Verlag, Berlin, 1994. Translated from the Russian by G. A. Yosifian [G. A. Iosif'yan].

[19] Alois Kufner and Anna-Margarete Sändig. Some applications of weighted Sobolev spaces, volume 100. BG Teubner Gmbh, 1987.

[20] William Charles Hector McLean. Strongly elliptic systems and boundary integral equations. Cambridge University Press, 2000.

[21] Benjamin Muckenhoupt. Weighted norm inequalities for the Hardy maximal function. Transactions of the American Mathematical Society, 165:207-226, 1972.

[22] François Murat and Luc Tartar. Calculus of variations and homogenization [ MR0844873 (87i:73059)]. In Topics in the mathematical modelling of composite materials, volume 31 of Progr. Nonlinear Differential Equations Appl., pages 139-173. Birkhäuser Boston, Boston, MA, 1997.

[23] François Murat and Luc Tartar. $H$-convergence. In Topics in the mathematical modelling of composite materials, volume 31 of Progr. Nonlinear Differential Equations Appl., pages 21-43. Birkhäuser Boston, Boston, MA, 1997.

[24] Amnon Pazy. Semigroups of linear operators and applications to partial differential equations, volume 44. Springer Science \& Business Media, 2012.

[25] A. Piatnitski and E. Zhizhina. Periodic homogenization of nonlocal operators with a convolution-type kernel. SIAM J. Math. Anal., 49(1):64-81, 2017.

[26] Rémi Rhodes and Vincent Vargas. Scaling limits for symmetric Itô-Lévy processes in random medium. Stochastic Process. Appl., 119(12):4004-4033, 2009.

[27] Xavier Ros-Oton. Nonlocal elliptic equations in bounded domains: a survey. Publ. Mat., $60(1): 3-26,2016$.

[28] Walter Rudin. Functional analysis. International Series in Pure and Applied Mathematics. McGraw-Hill, Inc., New York, second edition, 1991.

[29] Angkana Rüland. Unique continuation for fractional Schrödinger equations with rough potentials. Communications in Partial Differential Equations, 40(1):77-114, 2015.

[30] Nikola Sandrić. Homogenization of periodic diffusion with small jumps. J. Math. Anal. Appl., 435(1):551-577, 2016.

[31] Russell W. Schwab. Periodic homogenization for nonlinear integro-differential equations. SIAM J. Math. Anal., 42(6):2652-2680, 2010.

[32] R. T. Seeley. Complex powers of an elliptic operator. 1967 Singular Integrals (Proc. Sympos. Pure Math., Chicago, Ill., 1966) pp. 288-307 Amer. Math. Soc., Providence, $R I$. 
[33] Elias M. Stein. Singular integrals and differentiability properties of functions. Princeton Mathematical Series, No. 30. Princeton University Press, Princeton, N.J., 1970.

[34] Pablo Raúl Stinga and José Luis Torrea. Extension problem and Harnack's inequality for some fractional operators. Communications in Partial Differential Equations, 35(11):2092-2122, 2010.

[35] Luc Tartar. The general theory of homogenization, volume 7 of Lecture Notes of the Unione Matematica Italiana. Springer-Verlag, Berlin; UMI, Bologna, 2009. A personalized introduction.

[36] Hans Triebel. Function spaces in Lipschitz domains and on Lipschitz manifolds. characteristic functions as pointwise multipliers. Revista Matemática Complutense, 15(2):475$524,2002$.

[37] Alexander Ivanovich Tyulenev. Description of traces of functions in the Sobolev space with a Muckenhoupt weight. Proceedings of the Steklov Institute of Mathematics, 1(284):280-295, 2014.

[38] Schwab Russell W. Stochastic homogenization for some nonlinear integro-differential equations Communications in Partial Differential Equations, 38(2):171-198, 2013. 